TRANSACTIONS OF THE

AMERICAN MATHEMATICAL SOCIETY

Volume 352, Number 6, Pages 2619-2642

S 0002-9947(00)02574-5

Article electronically published on March 7, 2000

\title{
RIGIDITY OF COXETER GROUPS
}

\author{
STRATOS PRASSIDIS AND BARRY SPIELER
}

\begin{abstract}
Let $W$ be a Coxeter group acting properly discontinuously and cocompactly on manifolds $N$ and $M(\partial M=\emptyset)$ such that the fixed point sets of finite subgroups are contractible. Let $f:(N, \partial N) \rightarrow\left(M \times D^{k}, M \times S^{k-1}\right)$ be a $W$-homotopy equivalence which restricts to a $W$-homeomorphism on the boundary. Under an assumption on the three dimensional fixed point sets, we show that then $f$ is $W$-homotopic to a $W$-homeomorphism.
\end{abstract}

\section{INTRODUCTION}

A reflection on a connected manifold $M$ is a locally linear involution on $M$ whose fixed point set separates $M$ into two components. A group $W$, generated by a set of reflections on a manifold, is called a reflection group if $W$ acts effectively and properly discontinuously on $M$. The algebraic structure of reflection groups is very specific. The only relations in the group are induced by the intersections of the fixed submanifolds of the generators ([13; 30]). Groups with such algebraic structure are called Coxeter groups because they were studied first in a paper by H. S. M. Coxeter (12]).

More specifically, a Coxeter system $(W, S)$ is a group $W$ with a presentation

$$
W=\left\langle s \in S: s^{2}=\left(s s^{\prime}\right)^{m_{s s^{\prime}}}=1, s \neq s^{\prime}, m_{s s^{\prime}} \in\{2,3, \ldots, \infty\}\right\rangle .
$$

In this paper we are interested in finitely generated (and therefore finitely presented) Coxeter groups.

In geometric topology, reflection groups appeared in the work of M. Davis ([13]; 14]), in the construction of closed aspherical topological manifolds not covered by Euclidean spaces. Using Davis' methods, G. Moussong constructed contractible, complete, non-positively curved (in the sense of Gromov), topological manifolds which are not homeomorphic to $\mathbb{R}^{n}([25])$.

Let $W$ be a Coxeter group which admits a properly discontinuous, cocompact action on a manifold $M$ (without boundary) such that the fixed point sets of finite subgroups are non-empty and contractible. We do not assume that the generators of $W$ act by reflections. The main result (Theorem 5.3) of the paper is the topological rigidity of the $W$-action on $M$.

Received by the editors November 14, 1997.

1991 Mathematics Subject Classification. Primary 57S25, 57N70, 20F55, 57S30.

Key words and phrases. Coxeter groups, reflection groups, rigidity theorems, equivariant topological Whitehead group.

The first author was supported in part by Vanderbilt University Summer Research Fellowship, and by National Science Foundation Grant DMS-9504479.

(C)2000 American Mathematical Society 
Theorem (Main Theorem). Let $N$ be a manifold with boundary on which $W$ acts locally linearly, properly discontinuously and with the property that the fixed point sets of finite subgroups are non-empty and contractible. Let

$$
f:(N, \partial N) \rightarrow\left(M \times D^{k}, M \times S^{k-1}\right)
$$

be a $W$-homotopy equivalence which restricts to a $W$-homeomorphism on the boundary. If the three dimensional fixed point sets of $N$ and $M \times D^{k}$ can be embedded in $S^{3}$, then $f$ is $W$-homotopic to a $W$-homeomorphism.

We give an outline of the proof of the main theorem. The assumptions of the theorem imply that $W$ is a virtual Poincaré duality group. By the results in [16], it splits as a product $W_{1} \times W_{2}$, where $W_{1}$ is a a Coxeter group acting cocompactly by reflections on a contractible manifold and $W_{2}$ is a finite Coxeter group. The action of the group $W_{2}$ is trivial on all the spaces involved. Thus we can assume that $W$ is a Coxeter group that acts cocompactly by reflections on a contractible manifold $M^{\prime}$. Then the quotient space $Q^{\prime}$ (which can be identified with a fundamental domain of the action) is a manifold with boundary ([13]). The intersections $Q^{\prime} \cap M^{\prime s}=\partial Q^{\prime} \cap M^{\prime s}(s \in S)$, are manifolds with (possibly empty) boundary (13]), called panels or mirrors. The panels of $Q^{\prime}$ and their non-empty intersections are contractible manifolds with (possibly empty) boundary. Comparing with $M^{\prime}$ enables us to show that the assumptions on $M \times D^{k}$ and $N$ are sufficient to force $W$ to act by reflections on both manifolds. Then any $W$-map $f: N \rightarrow M \times D^{k}$ is $W$ homotopic to a map $g$ that restricts to a homotopy equivalence of the fundamental domains and preserves the panels. The map $g$ induces homotopy equivalence on each non-empty intersection of the panels which is a compact contractible manifold. Using induction on the dimension of the manifolds, we can assume that the map is already a homeomorphism on the boundary. The generalized Poincaré conjecture implies that the map is homotopic to a homeomorphism, relative the boundary. Repeating the induction process, we construct a panel preserving homotopy from the restriction of $g$ to a homeomorphism. Standard constructions outlined in [13] extend the homotopy already constructed to a $W$-homotopy from $g$ (and therefore from $f$ ) to a $W$-homeomorphism. The assumption on the 3 -dimensional fixed point sets is needed in the inductive process because the Poincaré conjecture is open in dimension 3. The result of our Main Theorem has been proved by E. Rosas ([26]) for right angled reflection groups (i.e. the exponents in the Coxeter relations are equal to either 2 or $\infty$ ).

The rigidity theorem for Coxeter groups proves the Borel-Quinn conjecture for Coxeter groups. The Borel-Quinn conjecture generalizes the rigidity phenomena expressed by the Borel conjecture to an equivariant setting. It has been formulated explicitly by Connolly and Koźniewski ([10]). Let $\Gamma$ be a virtually torsion free, cocompact, discrete subgroup of the group of isometries of a complete, non-positively curved topological manifold $\tilde{M}$. Let $\Gamma_{0}$ be a normal torsion free subgroup of finite index, and $G=\Gamma / \Gamma_{0}$ the finite quotient. Let $\tilde{M}^{\prime}$ be a contractible $\Gamma$-manifold. We assume that the actions of $\Gamma$ on $\tilde{M}$ and $\tilde{M}^{\prime}$ are properly discontinuous and cocompact, and that the fixed point set of each finite group of $\Gamma$ is a contractible manifold, flatly embedded in a bigger fixed point set. The assumptions imply that there is a $\Gamma$-homotopy equivalence $F: \tilde{M}^{\prime} \rightarrow \tilde{M}$. Let $M=\tilde{M} / \Gamma_{0}, M^{\prime}=\tilde{M}^{\prime} / \Gamma_{0}$ be the compact $G$-manifolds determined by the $\Gamma$ actions on $\tilde{M}, \tilde{M}^{\prime}$. Then the map $F$ induces a $G$-homotopy equivalence $f: M^{\prime} \rightarrow M$. We assume that the torsion of 
$f$ is zero in $W h_{G}^{T o p, \rho}(M)$ (the equivariant topological Whitehead group introduced in [28] and [29], for classifying isovariant $G$ - $h$-cobordisms over $M$ satisfying certain gap conditions).

Conjecture (Borel-Quinn Conjecture). With the preceding notation the $\Gamma$-map $F$ is $\Gamma$-homotopic to a $\Gamma$-homeomorphism.

When the conjecture was stated in [10, there were three types of assumptions imposed: assumptions on the restriction of $f$ to the fixed point sets of dimension less than or equal to 4, gap hypotheses, and assumptions on the finite subgroups of $\Gamma$ of even order. The assumptions were necessary because of the examples given in [11, [36]. In the case of Coxeter groups, the gap hypotheses and the assumptions of subgroups of even order are not needed. We do need a condition on the three dimensional fixed point sets which is weaker than the hypotheses imposed in 10.

The condition on the torsion of $f$ is a necessary condition. We will explain why the condition is not needed when $\Gamma=W$ is a Coxeter group acting on $M$ by reflections. Let $W_{0}$ be a normal subgroup of finite index in $W, G=W / W_{0}$, and $M_{0}=M / W_{0}$. In Section 5, we show that (Theorem 5.3)

$$
W h_{G}^{T o p, \rho}\left(M_{0} \times T^{n}\right)=0,
$$

where $T^{n}$ is the $n$-dimensional torus with the trivial $G$-action. The proof is an adjustment of the ideas used in the proof of the main theorem.

In Section 2, we review the basic algebraic theory of Coxeter groups and the basic theory of panel spaces associated to Coxeter systems.

In Section 3, we show that the action of a Coxeter group on a universal space has the property that the fixed point sets are contractible if and only if all the panels are contractible. In this section, we extend the basic technical construction in [26] from the case of right angled reflection groups to general Coxeter groups.

In Section 4, we review the general theory of Coxeter groups when they act by reflections on polyhedra and manifolds, and the properties of the panel spaces determined by the action.

In Sections 5 and 6 , we prove the topological rigidity theorem and the $K$-theory rigidity theorems for Coxeter groups, as outlined in the preceding paragraphs.

We would like to thank N. Cardim, R. Charney, F. Connolly, M. daSilva, M. Davis, B. Hughes, M. Mihalik and Pedro Ontaneda for their helpful suggestions during the preparation of this paper.

\section{Preliminaries on Coxeter Groups}

We review the basic properties of Coxeter groups. Basic references are [3], [24], and [5] for a more geometric approach.

A Coxeter system $(W, S)$ is a pair where $W$ is a group and $S$ is a set of generators of $W$, subject only to the relations

$$
\left(s s^{\prime}\right)^{m\left(s, s^{\prime}\right)}, m(s, s)=1, m\left(s, s^{\prime}\right)=m\left(s^{\prime}, s\right) \geq 2 \text { if } s \neq s^{\prime} .
$$

Thus $W$ is generated by a set of reflections, and the only relations in $W$ are induced by the angles of intersection between the hyperplanes corresponding to the reflections. The group $W$ is called a Coxeter group and the elements of $S$ are called simple reflections. The conjugates of $S$ are called reflections of the Coxeter system $(W, S)$. We say that a Coxeter system $(W, S)$ is the product of two Coxeter systems

$$
(W, S) \cong\left(W_{1}, S_{1}\right) \times\left(W_{2}, S_{2}\right)
$$


if $W \cong W_{1} \times W_{2}$ and $S_{i} \subset S, i=1,2$. A Coxeter system is called irreducible if it cannot written as a non-trivial product of Coxeter systems. We will consider finitely generated (and therefore finitely presented) Coxeter groups. For $w \in W, \ell(w)$ denotes the length of $w$ in the Coxeter presentation of $W$. The main properties of the length function in Coxeter groups are summarized in [24, Section 5.2.

Let $J \subseteq S$ and $W_{J}$ be the subgroup of $W$ generated by $J$. Then the pair $\left(W_{J}, J\right)$ is again a Coxeter system (3]; 24]). Subgroups of $W$ of the form $W_{J}(J \subseteq S)$ are called parabolic subgroups. Finite parabolic subgroups have the following universal property among the finite subgroups of $W$ ([30]; [26], Lemma 2.1; [4], Proposition 1.3):

Proposition 2.1. Let $H$ be a finite subgroup of $W$. Then there is a finite parabolic subgroup $W_{J}$ and an element $w \in W$ such that $H<w W_{J} w^{-1}$.

To a Coxeter system $(W, S)$ we associate a partially ordered set

$$
\mathcal{F}(W, S)=\left\{J \subseteq S:\left|W_{J}\right|<\infty\right\}
$$

ordered by set inclusion. The set $\mathcal{F}(W, S)$ is isomorphic to the partially ordered set of finite parabolic subgroups of $W$.

The study of Coxeter group actions on spaces is connected with the theory of spaces equipped with a panel structure. We review the general theory of panel spaces. The basic definitions and properties of panel spaces are contained in [13] and 34 .

Definition 2.1. An $S$-panel structure on a topological space $Q$ is a locally finite family of closed subspaces $\left(Q_{s}\right)_{s \in S}$, indexed by a set $S$. The subsets $Q_{s}$ are called panels of $Q$. The pair $\left(Q,\left(Q_{s}\right)_{s \in S}\right)$ is called an $S$-panel space.

For each $q \in Q$, we write $S(q)=\left\{s \in S: q \in Q_{s}\right\}$. For each non-empty subset $J \subseteq S$, set

$$
Q_{J}=\{q \in Q: J \subseteq S(q)\}=\bigcap_{s \in J} Q_{s}, \quad Q_{\sigma(J)}=\bigcup_{s \in J} Q_{s} .
$$

We define $Q_{\emptyset}=Q$. The formal boundary $Q$ is defined to be the union of all the panels:

$$
\delta Q=\bigcup_{s \in S} Q_{s}
$$

The subspaces $Q_{J}$ are called faces. We will consider panel structures with finitely many panels.

We fix a Coxeter system $(W, S)$. $S$-panel structures on spaces can be used for the construction of spaces equipped with a $W$-action. Let $\left(Q,\left(Q_{s}\right)_{s \in S}\right)$ be an $S$-panel space. Define

$$
\mathcal{U}(W, Q)=W \times Q / \sim
$$

where $(w, q) \sim\left(w^{\prime}, q^{\prime}\right)$ if and only if $q=q^{\prime}$ and $w^{-1} w^{\prime} \in W_{S(q)}$. The space $\mathcal{U}(W, Q)$, with the quotient topology, is called the universal space of the pair $(W, Q)$. We write $[w, q], w \in W, q \in Q$, for elements in $\mathcal{U}(W, Q)$. There is a natural embedding

$$
i: Q \rightarrow \mathcal{U}(W, Q), \quad q \rightarrow[e, q] .
$$

The group $W$ acts on $\mathcal{U}(W, Q)$ by left multiplication in the first coordinate. The isotropy group of the point $[e, q]$ is $W_{S(q)}$, because only the generators in $S(q)$ fix $[e, q]$. Therefore the isotropy group of a general element $[w, q]$ is $w W_{S(q)} w^{-1}$. The space $\mathcal{U}(W, Q)$ has the following universal property ([34], p. 1088). 
Proposition 2.2. For any $W$-space $M$ and any map $f: Q \rightarrow M$ such that $s f(q)=f(q)$, for all $s \in S, q \in Q_{s}$, there is a unique $W$-equivariant map $f^{*}$ : $\mathcal{U}(W, Q) \rightarrow M$ such that $f^{*}([e, q])=f(q)$.

Definition 2.2. Let $\left.\left(Q,\left(Q_{s}\right)_{s \in S}\right)\right)$ and $\left.\left(Q^{\prime},\left(Q_{s}^{\prime}\right)_{s \in S}\right)\right)$ be $S$-panel spaces. An $S$ panel map is a continuous function $f: Q \rightarrow Q^{\prime}$ that preserves panels, i.e., $f\left(Q_{s}\right) \subseteq Q_{s}^{\prime}$, for all $s \in S$.

We will apply Proposition 2.2 to panel maps. Let $f: Q \rightarrow Q^{\prime}$ be an $S$-panel map. Form the composition

$$
f^{\prime}: Q \stackrel{f}{\rightarrow} Q^{\prime} \stackrel{i^{\prime}}{\rightarrow} \mathcal{U}\left(W, Q^{\prime}\right),
$$

where $i^{\prime}$ is the natural inclusion. For $q \in Q_{s}$,

$$
s f^{\prime}(q)=s[e, f(q)]=[s, f(q)]=[e, f(q)]=f^{\prime}(q) .
$$

Thus $f^{\prime}$ induces a $W$-map

$$
\mathcal{U}(W, f): \mathcal{U}(W, Q) \rightarrow \mathcal{U}\left(W, Q^{\prime}\right) .
$$

Summarizing, we have

Corollary 2.3. Let $f: Q \rightarrow Q^{\prime}$ be an $S$-panel map. Then there is a unique $W$-map $\mathcal{U}(W, f): \mathcal{U}(W, Q) \rightarrow \mathcal{U}\left(W, Q^{\prime}\right)$ extending $f$.

The conclusion of Corollary 2.3 can be used in the formulation of the naturality properties of the universal construction. Thus panel homeomorphisms induce $W$ homeomorphisms on the classifying spaces. Also, panel homotopies, i.e., $S$-panel maps $F:\left(Q \times I,\left(Q_{s} \times I\right)_{s \in S}\right) \rightarrow\left(Q^{\prime},\left(Q_{s}^{\prime}\right)_{s \in S}\right)$, induce $W$-homotopies on the corresponding universal spaces.

Definition 2.3. Let $\left.\left(Q,\left(Q_{s}\right)_{s \in S}\right)\right)$ be an $S$-panel space. The panel structure on $Q$ is called $W$-finite if $S(q) \in \mathcal{F}(W, S)$ for each $q \in Q$.

The $W$-action on a classifying space $\mathcal{U}(W, Q)$ is proper if and only if the $S$-panel structure of $Q$ is $W$-finite (13], Lemma 13.4; 34, p. 1089).

Let $\left(Q,\left(Q_{s}\right)_{s \in S}\right)$ be an $S$-panel space with a $W$-finite structure, and let $\mathcal{U}(W, Q)$ denote the universal $(W, Q)$-space. We order the elements of $W$ so that $w_{1}=e$ and $w_{i}<w_{j}$ implies that $\ell\left(w_{i}\right) \leq \ell\left(w_{j}\right)$. Set

$$
T_{n}(Q)=\bigcup_{i=1}^{n} w_{i} Q \subseteq \mathcal{U}(W, Q) .
$$

Lemma 2.4. Let $n>1$. Then

$$
T_{n-1}(Q) \cap w_{n} Q=w_{n} Q_{\sigma\left(B\left(w_{n}\right)\right)},
$$

where $B\left(w_{n}\right)=\left\{s \in S: \ell\left(w_{n} s\right)<\ell\left(w_{n}\right)\right\}$. Furthermore, $B\left(w_{n}\right) \in \mathcal{F}(W, S)$ for every $n$.

Proof. When $\mathcal{U}(W, Q)$ is a manifold, the proof is given in [13], Section 8. The same proof applies to the general case.

For each $J \in \mathcal{F}(W, S), w \in W$, we can have a natural choice for the coset representatives for $w W_{J}$.

Lemma 2.5. Let $J \in \mathcal{F}(W, S)$. Then for any $w \in W$ there is a unique element $v \in w W_{J}$ of maximal length. Furthermore, $w$ is the element of maximal length in $w W_{J}$ if and only if $J \subseteq B(w)$. 
Proof. The element $v$ is the product of the minimal coset representative for $w W_{J}$ and the element of maximal length in $W_{J}$. The details appear in Lemma 1.7 in [16].

Definition 2.4. An $S$-panel space $\left(Q,\left(Q_{s}\right)_{s \in S}\right)$ is called an $S$-panel complex if $Q$ is a $\mathrm{CW}$-complex and all the faces are subcomplexes of $Q$.

For a Coxeter system $(W, S)$ there is a natural choice of a polyhedron equipped with a $W$-finite $S$-panel structure such that the faces are subpolyhedra. We write $K_{0}(W, S)$ for the abstract simplicial complex with vertex set $S$ and simplices the non-empty elements of $\mathcal{F}(W, S)$. Let $K(W, S)$ denote the cone of $K_{0}(W, S)$, i.e., $K(W, S)=K_{0}(W, S) \cup\{\emptyset\}$. We define an $S$-panel structure on the geometric realization $|K(W, S)|$ of the simplicial complex. The panel corresponding to $s \in S$ is the geometric realization of the closed star of $s$ in $K_{0}(W, S)([13])$.

We can now express the connection between certain homotopy properties of the panels of $Q$ and the classifying space $\mathcal{U}(W, Q)$.

Theorem 2.6. For an $S$-panel complex $\left(Q,\left(Q_{s}\right)_{s \in S}\right)$ the following are equivalent:

1. $\mathcal{U}(W, Q)$ is contractible.

2. $Q$ is contractible and $Q_{J}$ is acyclic for each $J \in \mathcal{F}(W, S)$.

3. The union of chambers $T_{n}(Q)$ is contractible for all $n \geq 1$.

Proof. When $\mathcal{U}(W, Q)$ is a manifold, the result is a special case of Theorem 10.1 and Corollary 10.3 in [13]. But the same methods can be applied to the general case.

Vinberg in 34, showed that every Coxeter group is isomorphic to a linear Coxeter group, i.e., a Coxeter group that is a subgroup of a general linear group and acts by reflections on a cone in a vector space. Using Selberg's lemma we conclude that Coxeter groups are virtually torsion free, i.e. they contain a torsion free subgroup of finite index. Actually a Coxeter group $(W, S)$ has finite virtual cohomological dimension

$$
\operatorname{vcd}(W) \leq \operatorname{dim}|K(W, S)|
$$

More precise statements can be found in 1], 18, and [22].

\section{Universal Spaces of Coxeter Systems}

Let $(W, S)$ be a Coxeter system and $\left(Q,\left(Q_{s}\right)_{s \in S}\right)$ a $W$-finite $S$-panel complex. The main purpose of this section is the proof that $\mathcal{U}(W, Q)$ is a $W$-space of type $\mathcal{E} W$ if and only if $Q$ and each face of $Q$ are contractible.

We start with a basic definition ([9], 27]).

Definition 3.1. Let $W$ be any discrete group. A space of type $\mathcal{E} W$ is a $W$-CWcomplex such that the isotropy groups of the action are finite, and the fixed point sets of finite subgroups are non-empty and contractible. The space $\mathcal{E} W$ is unique up to $W$-homotopy ([9]).

We formalize the $S$-panel structures that we will need.

Definition 3.2. An $S$-panel structure on a space $Q$ is called admissible if the panel structure is $W$-finite and $Q$ and each non-empty face are contractible. It is called an admissible $S$-panel complex structure, if in addition, $Q$ is an $S$-panel complex. 
Let $K(W)$ be the $W$-complex constructed in [25. More explicitly, $K(W)$ is defined as the universal space of the $S$-panel space $C\left|K_{0}(W, S)^{\prime}\right|$, the cone on the geometric realization of the first barycentric subdivision of $K_{0}(W, S)$. The panel corresponding to $s$ is the geometric realization of the closed star of $s$ in $K_{0}(W, S)^{\prime}$. Thus $C\left|K_{0}(W, S)^{\prime}\right|$ is an admissible complex, by construction, and $K(W)=\mathcal{U}\left(W, C\left|K_{0}(W, S)^{\prime}\right|\right)$. Also, $K(W)$ admits a locally flat metric of nonpositive curvature such that $W$ acts by isometries and reflections, i.e. the fixed point set of each generator $s \in S$ separates $K(W)$ in two components.

The authors are grateful to M. Davis for bringing to their attention the following result, which is an immediate consequence of the properties of the non-positive curvature metric on $K(W)$.

Lemma 3.1. The $W$-complex $K(W)$ is a complex of type $\mathcal{E} W$.

Proof. The action of $W$ on $K(W)$ is proper, so the isotropy groups of the action are finite. We have to show that fixed point sets of finite subgroups are contractible. This is a general statement about the fixed point sets of groups of isometries of complexes of non-positive curvature. Let $H$ be a finite subgroup of $W$. Let $x, y$ be two points fixed by $H$. Then there is a unique geodesic $\alpha$ of minimal length joining $x$ and $y$. Since $H$ acts by isometries, $\alpha$ is a geodesic in $K(W)^{H}$. In [15], Proposition 1.4, a contraction $c: K(W) \times I \rightarrow K(W)$ is defined by contracting along the geodesics. Therefore $c$ restricts to a contraction on $K(W)^{H}$.

Lemma 3.2. Let $\left(Q,\left(Q_{s}\right)_{s \in S}\right)$ be an admissible $S$-panel space such that $\left(Q_{J}, \delta Q_{J}\right)$ satisfies the homotopy extension property for each $J \in \mathcal{F}(W, S)$. Then $\mathcal{U}(W, Q)$ is $W$-homotopy equivalent to $K(W)$. In particular, if $\left(Q,\left(Q_{s}\right)_{s \in S}\right)$ is an admissible $S$-complex, $\mathcal{U}(W, Q)$ is a complex of type $\mathcal{E} W$.

Proof. By Proposition 2.5 in [26], there is an $S$-panel map $f: Q \rightarrow C\left|K_{0}(W, S)^{\prime}\right|$. Since all the faces in both complexes are contractible, $f$ is an $S$-panel homotopy equivalence. The functorial properties of the universal construction (Corollary 2.3) imply that:

$$
\mathcal{U}(W, f): \mathcal{U}(W, Q) \rightarrow K(W)
$$

is a $W$-homotopy equivalence.

We will show that the converse of Lemma 3.2 is true. We start with a $W$-finite $S$-panel complex $Q$ such that the universal space $U=\mathcal{U}(W, S)$ is a space of type $\mathcal{E} W$, and show that $Q$ is an admissible complex.

Let $J \in \mathcal{F}(W, S)$. We will describe $U^{W_{J}}$, the fixed point set of $W_{J}$, as a union of faces. We fix a total ordering of $W$ as in Section 2.

Definition 3.3. With the above notation:

1. Let $w \in W, K \in \mathcal{F}(W, S)$. We say that the pair $(w, K)$ represents a face in $U^{W_{J}}$ if $J \subset w W_{K} w^{-1}$.

2. An element $v \in W$ is called J-maximal in the pair $(w, K)$ if $(w, K)$ represents a face of $U^{W_{J}}$ and $v$ is the unique maximal element in the coset $w W_{K}$ (Lemma 2.5).

We write

$$
V_{J}=\{(v, K): \exists w \in W, v \text { is the } J \text {-maximal element in }(w, K)\} .
$$

Define a total ordering on the set $V_{J}$ as follows: $(v, K) \prec\left(v^{\prime}, K^{\prime}\right)$ if and only if 
1. $v<v^{\prime}$ in the total ordering of $W$, and

2. if $v=v^{\prime}, K \supsetneqq K^{\prime}$.

Remark 3.1. We summarize the obvious properties of the ordering.

1. The minimal element in the ordering is the pair $\left(w_{J}, J\right)$, where $w_{J}$ is the unique element of maximal length in $W_{J}$.

2. Lemma 2.5 implies that $v$ is $J$-maximal in $(v, K)$ if and only if $K \subseteq B(v)$.

Our goal is to describe the structure of $U^{W_{J}}$ written as the union of faces indexed by $V_{J}$.

Lemma 3.3. The space $U^{W_{J}}$ can be written as union of faces $v Q_{K}$ such that $v$ is $J$-maximal in the pair $(w, K)$.

Proof. There are two immediate consequences of the definitions.

1. If $(w, K)$ is a pair representing a face in $U^{W_{J}}$, then $w Q_{K} \subseteq U^{W_{J}}$.

2. If $v$ is $J$-maximal in $(w, K)$, then $v Q_{K}=w Q_{K}$.

These observations imply that each face $v Q_{K}$, with $v$ the $J$-maximal element in $(w, K)$ is contained in $U^{W_{J}}$.

For the other inclusion, let $x \in U^{W_{J}}$. Then the isotropy group of $x$ is a conjugate of a parabolic subgroup, i.e., $W_{x}=w W_{K} w^{-1}$ for some $K \in \mathcal{F}(W, S), w \in W$. Since $x$ is fixed by $W_{J}$, it follows that $J \subset w W_{K} w^{-1}$ and the pair $(w, K)$ represents a face in $U^{W_{J}}$. Thus $x \in w Q_{K}=v Q_{K}$, where $v$ is the $J$-maximal element in $(w, K)$.

Imitating the construction in Section 2, we define

$$
T_{(v, K)}^{J}=\bigcup_{\left(v^{\prime}, K^{\prime}\right) \preceq(v, K)} v^{\prime} Q_{K^{\prime}} .
$$

Definition 3.4. An element $(v, K)$ of $V_{J}$ is called reducible if $v Q_{K} \subseteq T_{\left(v^{\prime}, K^{\prime}\right)}^{J}$ for some $\left(v^{\prime}, K^{\prime}\right) \supsetneqq(v, K)$. Otherwise $(v, K)$ is called irreducible.

Remark 3.2. The pair $(v, B(v))$ is never irreducible, because, for any $K \varsubsetneqq B(v)$, $(v, K) \supsetneqq(v, B(v))$ and $v Q_{B(v)} \varsubsetneqq v Q_{K}$.

The following result is the analogue of Lemma 2.4.

Lemma 3.4. Let $(v, K) \in V_{J}-\left\{\left(w_{J}, J\right)\right\}$, and let $\left(v^{\prime}, K^{\prime}\right)$ denote the maximal element in $V_{J}$ smaller than $(v, K)$. If $(v, K)$ is irreducible, then

$$
v Q_{K} \cap T_{\left(v^{\prime}, K^{\prime}\right)}^{J}=\bigcup_{s \in B(v)-K} v Q_{K \cup\{s\}} .
$$

Proof. First notice that, for all $s \in B(v), K \cup\{s\} \in \mathcal{F}(W, S)$ by part (2) of Remark 3.1 Thus $Q_{K \cup\{s\}} \neq \emptyset$.

We start with the inclusion

$$
\bigcup_{s \in B(v)-K} v Q_{K \cup\{s\}} \subseteq v Q_{K} \cap T_{\left(v^{\prime}, K^{\prime}\right)}^{J}
$$

For each $s \in B(v)-K, v Q_{K \cup\{s\}} \subseteq v Q_{K}$. Since $K \cup\{s\} \subseteq B(v)$, Lemma 2.5 implies that $v$ is $J$-maximal in $(v, K \cup\{s\})$. Then $(v, K \cup\{s\}) \supsetneqq(v, K)$ and $v Q_{K \cup\{s\}} \subseteq$ $T_{\left(K^{\prime}, v^{\prime}\right)}^{J}$.

For the other inclusion, let $v x \in v Q_{K} \cap T_{\left(K^{\prime}, v^{\prime}\right)}^{J}$. Then there is $\left(v^{\prime \prime}, K^{\prime \prime}\right) \supsetneqq(v, K)$ such that $v x \in v Q_{K} \cap v^{\prime \prime} Q_{K^{\prime \prime}}$. Then the intersection $Q_{K} \cap v^{-1} v^{\prime \prime} Q_{K^{\prime \prime}}$ is a non-empty face. If $Q_{K} \cap v^{-1} v^{\prime \prime} Q_{K^{\prime \prime}}=Q_{K}$, then $v Q_{K} \subseteq v^{\prime \prime} Q_{K^{\prime \prime}} \subset T_{\left(K^{\prime}, v^{\prime}\right)}^{J}$, contradicting the assumption that $(v, K)$ is irreducible. Thus $x$ must belong to a proper subface of $Q_{K}$. We distinguish two cases: 
1. $v^{\prime \prime}<v$ in the ordering of $W$. Let $v=w_{n}$ in the ordering of $W$. By assumption, $x \in v Q_{K} \cap T_{n-1}(Q)$. By Lemma 8.2 in [13], $x \in \bigcup_{s \in B(v)} Q_{s}$. Since $x$ belongs to a proper subface of $Q_{K}$, there must be $s \in B(v)-K$ such that $x \in Q_{K \cup\{s\}}$.

2. $v=v^{\prime \prime}, K \varsubsetneqq K^{\prime \prime}$. Since $v$ is a $J$-maximal element in $\left(v, K^{\prime \prime}\right), K^{\prime \prime} \subseteq B(v)$. If $s \in K^{\prime \prime}-K$ is a simple reflection, then

$$
v x \in v Q_{K} \cap v Q_{K^{\prime \prime}}=v Q_{K^{\prime \prime}} \subseteq v Q_{K \cup\{s\}} .
$$

Lemma 3.5. Let $\left(Q,\left(Q_{s}\right)_{s \in S}\right)$ be a $W$-finite $S$-panel complex such that $\mathcal{U}(W, Q)$ is a complex of type $\mathcal{E} W$. Then the $S$-panel structure of $Q$ is admissible.

Proof. Let $U=\mathcal{U}(W, Q)$ as before. By assumption $U$ is contractible, and by Theorem 10.1 in [13], $Q$ is contractible and each face $Q_{J}, J \in \mathcal{F}(W, S)$, is acyclic. We will show that $Q_{J}$ is contractible by reverse induction on the number of elements of $J$. If $J$ is a maximal subset in $\mathcal{F}(W, S)$, then $Q_{J}=U^{W_{J}}$, which is contractible by assumption. If $J$ is not maximal, then we assume that $Q_{K}$ is contractible for each $K \in \mathcal{F}(W, S),|J|<|K|$, and we will show that $Q_{J}$ is contractible. By Lemma 3.4 $U^{W_{J}}$ is given as an ascending union

$$
U^{W_{J}}=\bigcup_{(v, K) \in V_{J}} T_{(v, K)}^{J} .
$$

Fix an element $(v, K) \in V_{J}$. Let $\left(v^{\prime}, K^{\prime}\right)$ be the maximal element smaller than $(v, K)$. If $(v, K)$ is reducible, then $T_{(v, K)}^{J}=T_{\left(v^{\prime}, K^{\prime}\right)}^{J}$. If not, then $K \varsubsetneqq B(v)$ (Remark 3.2), and $v Q_{K} \cap T_{\left(v^{\prime}, K^{\prime}\right)}^{J}$ is the union of faces of the form $v Q_{K \cup\{s\}}, s \in B(v)-K$. By assumption, all the faces $v Q_{K \cup\{s\}}$ are contractible. Their intersection

$$
\bigcap_{s \in B(v)-K} v Q_{K \cup\{s\}}=v Q_{B(v)}
$$

is also contractible by the induction hypothesis $(|J| \leq|K| \supsetneqq|B(v)|)$. By Van Kampen's theorem, the map induced by inclusion, $\pi_{1}\left(T_{\left(v^{\prime}, K^{\prime}\right)}^{J}\right) \rightarrow \pi_{1}\left(T_{(v, K)}^{J}\right)$, is an injection. Thus the inclusion induced map $\pi_{1}\left(Q_{J}\right) \rightarrow \pi_{1}\left(U^{W_{J}}\right)$ is an injection $\left(Q_{J}=w_{J} Q_{J}\right.$ corresponds to the minimal element in $\left.V_{J}\right)$. Therefore $\pi_{1}\left(Q_{J}\right)=0$ and $Q_{J}$ is contractible.

Summarizing, we get

Proposition 3.6. The classifying space $\mathcal{U}(W, Q)$ of a finite $S$-panel complex $Q$ is of type $\mathcal{E} W$ if and only if $Q$ is admissible.

\section{Reflection Groups}

We study properties of groups that act by reflections on manifolds. It turns out that all such groups are Coxeter groups ([13]; [30]). In that context, algebraic properties of the group can help in determining geometric properties of the action. By 'manifold' we mean 'topological manifold'.

Definition 4.1. Let $M$ be a connected manifold, possibly with boundary.

1. A reflection on $M$ is a locally smooth involution $r: M \rightarrow M$ with fixed point set $M^{r}$ a submanifold of $M$ that separates $M$ into two components.

2. A discrete group $W$ that acts on $M$ effectively, properly discontinuously, and locally smoothly, and is generated by a set of reflections on $M$, is called a reflection group on $M$. 
Let $W$ be a reflection group on a connected manifold $M^{n}$. Then the action determines a set $S$ of Coxeter generators of $W$, and the elements of $W$ that act by reflections on $M$ are exactly the reflections in the Coxeter system $(W, S)([13)$. Furthermore, the action determines an $S$-panel space $\left(Q,\left(Q_{s}\right)_{s \in S}\right)$ ([13]). Actually $Q$, as a panel space, is modeled on the standard cone in $\mathbb{R}^{n}([13])$. Such a panel space $Q$ is called a panel manifold. If furthermore $\left(Q,\left(Q_{s}\right)_{s \in S}\right)$ is admissible it is called an admissible $S$-panel manifold.

Remark 4.1. The proofs given in [13] are for manifolds without boundary, but the same methods work when $W$ acts by reflections on a manifold with boundary. Let $Q$ be a chamber of such an action. Then $Q$ is an $S$-panel manifold, and it is also a manifold with boundary

$$
\partial Q=\delta Q \cup(Q \cap \partial M) .
$$

Also, each panel $Q_{s}$ is an $(S-\{s\})$-panel $(n-1)$-manifold. Inductively, each $Q_{J}$ is an $(S-J)$-panel $n-|J|$-manifold with boundary

$$
\partial Q_{J}=\bigcup\left(\left\{Q_{K}: J \varsubsetneqq K,|K|=|J|+1\right\}\right) \cup\left(Q_{J} \cap \partial M\right) .
$$

Definition 4.2. A Coxeter $W$-manifold is a contractible $W$-manifold (possibly with boundary) on which $W$ acts properly discontinuously, locally smoothly, cocompactly and so that the fixed point sets of finite subgroups are non-empty and contractible. In particular, a Coxeter $W$-manifold is a manifold of type $\mathcal{E} W$ ([9]; [27]).

Using the theory of reflection groups on manifolds, M. Davis characterized the Coxeter systems that admit cocompact actions, by reflections, on contractible manifolds. They are the Coxeter systems such that $K_{0}(W, S)$ is a generalized homology sphere, i.e., $K_{0}(W, S)$ and the link of each simplex are homology spheres. M. Davis' result concerns topological manifolds. There is a discussion for an extension of the results to smooth manifolds in Section 17, 13.

Definition 4.3. A Coxeter system $(W, S)$ is called a manifold-reflection system if $K_{0}(W, S)$ is a generalized homology sphere. The group $W$ is called a manifoldreflection group.

Remark 4.2. 1. For a manifold-reflection system $(W, S)$, the universal space $\mathcal{U}(W,|K(W, S)|)$ is a contractible $W$-manifold ([13) and a space of type $\mathcal{E} W$ (Proposition 3.6). Thus $\mathcal{U}(W,|K(W, S)|)$ is a Coxeter $W$-manifold.

2. In [16], the complete characterization of the virtual Poincaré duality Coxeter groups is given. It is shown that $(W, S)$ is a virtual Poincaré duality group if and only $(W, S) \cong\left(W_{1}, S_{1}\right) \times\left(W_{2}, S_{2}\right)$, where $W_{2}$ is a finite Coxeter group and $\left(W_{1}, S_{1}\right)$ is a manifold-reflection system.

In the following lemma we characterize the action of a reflection group on Coxeter manifolds.

Lemma 4.1. With the above notation:

1. Let $M$ be a Coxeter $W$-manifold without boundary and $(W, S)$ a manifoldreflection group. Then $W$ acts by reflections on $M$.

2. Let $\left(M_{i}^{n}, \partial M_{i}\right), i=1,2$, be Coxeter $W$-manifolds with boundary. Let $W$ be a manifold-reflection group that acts by reflections on $M_{2}$ and $\partial M_{2}$. Let

$$
f:\left(M_{1}, \partial M_{1}\right) \rightarrow\left(M_{2}, \partial M_{2}\right)
$$


be a W-homotopy equivalence which restricts to a W-homeomorphism on $\partial M_{1}$. Then $W$ acts by reflections on $M_{1}$.

Furthermore, an element of $W$ acts as a reflection on $M_{1}$ if and only if it acts as a reflection on $M_{2}$.

Proof. (i) In [26], Proposition 2.14 and Proposition 3.8, the result is proved for $W$ a right angled Coxeter group. The proof extends to the general case. The proof actually shows that a reflection of $(W, S)$ acts as a reflection on $M$.

(ii) Let $S$ be a set of Coxeter generators induced by the $W$-action on $M_{2}$ (Theorem 2.1). Let $r \in W$ be an element that acts as a reflection. First notice that $M_{1}^{r}$ has the same dimension as $M_{2}^{r}$ because their boundaries have the same dimension. Thus $M_{1}^{r}$ is codimension 1 submanifold of $M_{1}$. Let $M^{\prime}{ }_{i}=M_{i}-\partial M_{i}, i=1,2$. Then $f$ induces a proper $W$-homotopy equivalence from $M^{\prime}{ }_{1}$ to $M^{\prime}{ }_{2}$. Thus

$$
H_{c}^{n}\left(M_{1}^{\prime}, M_{1}^{\prime r}\right) \cong H_{c}^{n}\left(M_{2}^{\prime}, M_{2}^{\prime r}\right)
$$

and Alexander-Poincaré duality implies that

$$
H_{0}\left(M_{1}^{\prime}-M_{1}^{\prime r}\right) \cong H_{0}\left(M_{2}^{\prime}-M_{2}^{\prime r}\right) \cong \mathbb{Z} \oplus \mathbb{Z}
$$

because $s$ acts as a reflection on $M^{\prime}{ }_{2}$. So $M_{1}^{\prime r}$ is a codimension one submanifold of $M^{\prime}{ }_{1}$ that separates $M^{\prime}{ }_{1}$ into two components. Thus $W$ is generated by a set of elements that act by reflections on $M^{\prime}{ }_{1}$ and $\partial M_{1}$. Therefore $W$ acts by reflections on $M_{1}$.

The same argument shows that if $r \in W$ acts as a reflection on $M_{1}$, then it acts as a reflection on $M_{2}$.

Notice that, in both cases, the $W$-action on $M_{1}$ is effective because the action on $M_{2}$ is effective.

The following lemma holds for any discrete group $W$.

Lemma 4.2. Let $M$ be a contractible manifold on which $W$ acts properly discontinuously and cocompactly. Let $W=W_{1} \times W_{2}$ be a decomposition of $W$ such that $W_{2}$ is finite and $M^{W_{2}}$ is contractible. If $\partial M \neq \emptyset$, assume that $W_{2}$ acts trivially on $\partial M$. Then $W_{2}$ acts trivially on $M$.

Proof. We first assume that $\partial M=\emptyset$. The group $N\left(W_{2}\right) / W_{2} \cong W_{1}$ acts on the fixed point set $M^{W_{2}}$. Since $M^{W_{2}} / W_{1}$ is a closed subset of the compact space $M / W_{1}$, the action of $W_{1}$ on the contractible manifold (without boundary) $M^{W_{2}}$ is cocompact. Thus

$$
\operatorname{dim}\left(M^{W^{2}}\right)=\operatorname{vcd}\left(W_{1}\right)=\operatorname{vcd}(W)=\operatorname{dim}(M) .
$$

Therefore $M^{W_{2}}$ is a submanifold without boundary of the connected manifold $M$ of the same dimension, which is closed as a topological subspace. By the invariance of domain, $M^{W_{2}}=M$, which implies that $W_{2}$ acts trivially on $M$.

If $\partial M \neq \emptyset$, then the fixed point set $M^{W_{2}}$, is a submanifold of $M$ with boundary $\partial M$ which is a closed subspace. As before, $M^{W_{2}}=M$ and $W_{2}$ acts trivially.

Corollary 4.3. Let $(W, S)$ be a manifold-reflection group. Then there is no decomposition of $(W, S)$ as a product $\left(W_{1}, S_{1}\right) \times\left(W_{2}, S_{2}\right)$ with $W_{2}$ a finite Coxeter group.

Proof. Let $M$ be a contractible manifold on which $W$ acts by reflections. Thus $W_{2}$ acts by reflections on $M$, which contradicts Lemma 4.2 
We will now study uniqueness properties of the Coxeter presentation of a manifoldreflection Coxeter group.

Lemma 4.4. Let $(W, S)$ be a manifold-reflection system. Then $W_{S-\{s\}}$ has infinite index in $W$ for each $s \in S$. Furthermore, $\operatorname{vcd}\left(W_{S-\{s\}}\right)<\operatorname{vcd}(W)$.

Proof. We will give a proof by contradiction. We assume that $W_{S-\{s\}}$ has finite index in $W$. The Coxeter system $(W, S)$ can be written as a product of Coxeter systems $\left(W_{i}, S_{i}\right), i=1, \ldots k$, such that, for each $i,\left(W_{i}, S_{i}\right)$ is irreducible. Let $s \in S_{i_{0}}$. Then there is a bijection between right cosets $W / W_{S-\{s\}}$ and $W_{S_{i_{0}}} / W_{S_{i_{0}}-\{s\}}$. Thus $\left[W_{S_{i_{0}}}: W_{S_{i_{0}}-\{s\}}\right]<\infty$. Proposition 4.2(ix) in [17] implies that $W_{S_{i_{0}}}$ is finite, contradicting Corollary 4.3 .

Since $W$ is a virtual Poincaré duality group, it contains a Poincaré duality subgroup $W_{0}$ of finite index. Then the subgroup $W_{0} \cap W_{S-\{s\}}$ is a torsion free subgroup of finite index of $W_{S-\{s\}}$, and it is contained in $W_{0}$. By [31], $\operatorname{cd}\left(W_{0} \cap W_{S-\{s\}}\right)<$ $\operatorname{cd}\left(W_{0}\right)$. But

$$
\operatorname{vcd}\left(W_{S-\{s\}}\right)=\operatorname{cd}\left(W_{0} \cap W_{S-\{s\}}\right)<\operatorname{cd}\left(W_{0}\right)=\operatorname{vcd}(W) .
$$

Let $(W, S)$ be a Coxeter system which acts on a manifold $M^{n}$ by reflections. We write $Q$ for the fundamental domain, which is homeomorphic to a manifold with boundary. Write

$$
R=\bigcup_{w \in W} w S w^{-1} .
$$

Then the elements of $R$ act on $M$ by reflections, and the singular set of the action is given by

$$
\sigma(M)=\bigcup_{r \in R} M^{r}
$$

In what follows, $\mathbb{Z} / 2 \mathbb{Z}$-coefficients are understood. There is a Poincaré duality isomorphism

$$
P: H_{c}^{n}(M, \sigma(M)) \rightarrow H_{0}(M-\sigma(M)) .
$$

But since the action is by reflections,

$$
M-\sigma(M)=\bigcup_{w \in W} \operatorname{int}(w Q) .
$$

By the naturality of the Poincaré duality, the inclusion maps induce an isomorphism

$$
\iota_{c}^{*}=\bigoplus_{w \in W} \iota_{w}^{*}: H_{c}^{n}(M, \sigma(M)) \rightarrow \bigoplus_{w \in W} H_{c}^{n}(w Q, \partial(w Q)) .
$$

Thus the set $\mathcal{B}=\left\{\left(i_{c}^{*}\right)^{-1}\left([w Q, \partial(w Q)]^{*}\right) / w \in W\right\}$ forms a basis of $H_{c}^{n}(M, \sigma(M))$ (here $[w Q, \partial(w Q)]^{*}$ demotes the dual of the Poincaré class of $(w Q, \partial(w Q))$. We write $[w Q]^{*}$ for $\left(\iota_{c}^{*}\right)^{-1}\left([w Q, \partial(w Q)]^{*}\right)$. On the other hand, $W$ acts on $H_{c}^{n}(M, \sigma(M))$, on the left, in such a way that each element $w$ acts through the map

$$
\left(w^{-1}\right)^{*}: H_{c}^{n}(M, \sigma(M)) \rightarrow H_{c}^{n}(M, \sigma(M)) .
$$

Under this action, $H_{c}^{n}(M, \sigma(M))$ has the structure of a free left $\mathbb{Z} / 2 \mathbb{Z}[W]$-module on one generator $[Q]^{*}$.

Lemma 4.5. Let $W=\langle r\rangle \cong \mathbb{Z} / 2 \mathbb{Z}$ act by reflections on manifolds $M_{i}^{n}, i=1,2$, and let $\mathcal{B}_{i}, i=1,2$, be the $\mathbb{Z} / 2 \mathbb{Z}$-bases of $H_{c}^{n}\left(M_{i}, M_{i}^{r}\right)$ corresponding to the components of $M_{i}-M_{i}^{r}$. If $f: M_{1} \rightarrow M_{2}$ is a proper $W$-homotopy equivalence, then $f^{*}\left(\mathcal{B}_{2}\right)=\mathcal{B}_{1}$. 
Proof. Identify $H_{c}^{n}\left(M_{i}, M_{i}^{r}\right)$ with the free $\mathbb{Z} / 2 \mathbb{Z}[W]$-module with basis a fundamental domain $\left[Q_{i}\right](i=1,2)$. Then the map $f^{*}: H_{c}^{n}\left(M_{2}, M_{2}^{r}\right) \rightarrow H_{c}^{n}\left(M_{1}, M_{1}^{r}\right)$ is a $\mathbb{Z} / 2 \mathbb{Z}[W]$-isomorphism. Thus it is given by multiplication by a unit of $\mathbb{Z} / 2 \mathbb{Z}[W]$. But the units of $\mathbb{Z} / 2 \mathbb{Z}[W]$ are $\{1, r\}$. Then $f^{*}\left(\left[Q_{2}\right]\right)$ is equal to $\left[Q_{1}\right]$ or to $r^{*}\left(\left[Q_{1}\right]\right)$, which implies the result.

We assume further that the action of $W$ on $M$ is cocompact. In this case, all the domains $w Q$ are compact manifolds with boundary. There is an isomorphism ([19], Ch. VIII, §2)

$$
J: H_{n}(M, \sigma(M)) \rightarrow \Gamma_{c}(M-\sigma(M)),
$$

where the term on the right is the vector space generated by the components of $M-\sigma(M)$ that have compact closure. Since all the components have compact closure, the inclusion maps induce an isomorphism

$$
\iota_{*}=\bigoplus_{w \in W} \iota_{w *}: \bigoplus_{w \in W} H_{n}(w Q, \partial(w Q)) \rightarrow H_{n}(M, \sigma(M)) .
$$

For each $w \in W$ we have $w_{*}: H_{n}(M, \sigma(M)) \rightarrow H_{n}(M, \sigma(M))$, and the collection of the maps $w_{*}$ induces an action of $W$ on $H_{n}(M, \sigma(M))$. Under this action, $H_{n}(M, \sigma(M))$ becomes a free left $\mathbb{Z} / 2 \mathbb{Z}[W]$-module with generator $[Q]$, the image of the fundamental class of $(Q, \partial(Q))$.

We also consider the dual construction. Taking duals, as $\mathbb{Z} / 2 \mathbb{Z}$ vector spaces, results to an isomorphism

$$
\prod_{w \in W} \iota_{w}^{*}: H^{n}(M, \sigma(M)) \rightarrow \prod_{w \in W} H^{n}(w Q, \partial(w Q)) .
$$

The induced action of $W$ on $H^{n}(M, \sigma(M))$ is by the formula $x \cdot w=w^{*}(x)$ and induces a right $\mathbb{Z} / 2 \mathbb{Z}[W]$-module structure on it. Since $\mathbb{Z} / 2 \mathbb{Z}[W]$ is a ring with involution, $\left.H^{n}(M, \sigma(M))\right)$ admits a natural left $\mathbb{Z} / 2 \mathbb{Z}[W]$-module structure with $w \cdot x=x \cdot w^{-1}=\left(w^{-1}\right)^{*}(x)$. We would like to characterize the dual of $H_{n}(M, \sigma(M))$ as a $\mathbb{Z} / 2 \mathbb{Z}[W]$-module. By [6], Ch. VIII, Lemma 7.4,

$$
\operatorname{Hom}_{\mathbb{Z} / 2 \mathbb{Z}[W]}\left(H_{n}(M, \sigma(M)), \mathbb{Z} / 2 \mathbb{Z}[W]\right) \cong \operatorname{Hom}_{c}\left(H_{n}(M, \sigma(M)), \mathbb{Z} / 2 \mathbb{Z}\right),
$$

where the last group consists of $\mathbb{Z} / 2 \mathbb{Z}$-homomorphisms $\phi$ from $H_{n}(M, \sigma(M))$ to $\mathbb{Z} / 2 \mathbb{Z}$ such that $\phi(w x)=0$ for all but finitely many $w$. In this case,

$$
\operatorname{Hom}_{c}\left(H_{n}(M, \sigma(M)), \mathbb{Z} / 2 \mathbb{Z}\right) \cong \bigoplus_{w \in W} H^{n}(w Q, \partial(w Q))
$$

as left $\mathbb{Z} / 2 \mathbb{Z}[W]$-modules. But there is a Poincaré duality isomorphism

$$
P: H_{c}^{n}(M, \sigma(M)) \rightarrow H_{0}(M-\sigma(M))
$$

which implies that

$$
\iota_{c}^{*}=\bigoplus_{w \in W} \iota_{w}^{*}: H_{n}^{c}(M, \sigma(M)) \rightarrow \bigoplus_{w \in W} H^{n}(w Q, \partial(w Q))
$$

is an isomorphism. Thus the $\mathbb{Z} / 2 \mathbb{Z}[W]$-dual of $H_{n}(M, \sigma(M))$ is isomorphic to $H_{c}^{n}(M, \sigma(M))$, and the following diagram commutes:

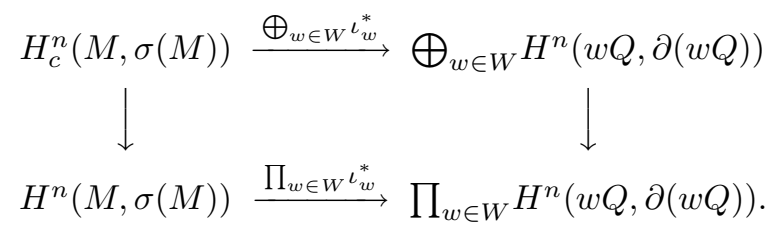

where the vertical maps are the natural maps. 
Combining the above remarks, we get

Lemma 4.6. The $\mathbb{Z} / 2 \mathbb{Z}[W]$-vector spaces $H_{n}(M, \sigma(M))$ and $H_{c}^{n}(M, \sigma(M))$ are free $\mathbb{Z} / 2 \mathbb{Z}[W]$-modules which are naturally dual to each other, i.e., if $M$ and $N$ are two manifolds equipped with a $W$-action by reflections and $f: M \rightarrow N$ is a proper $W$-map, then $f^{*}: H_{c}^{n}(N, \sigma(N)) \rightarrow H_{c}^{n}(M, \sigma(M))$ is the $\mathbb{Z} / 2 \mathbb{Z}[W]$-dual of the map $f_{*}: H_{n}(M, \sigma(M)) \rightarrow H_{n}(N, \sigma(N))$.

Proof. . The first part follows from the remarks before the statement of the lemma. For the naturality property, first notice that the map induced on cohomology is the $\mathbb{Z} / 2 \mathbb{Z}$-dual of $f_{*}$. The result follows by considering the cohomology groups with compact supports as subgroups of the ordinary cohomology and using the commutativity of the last diagram.

Proposition 4.7. Let $M_{i}^{n}, i=1,2$, be Coxeter $W$-manifolds without boundary. Let $W$ act by reflections on $M_{2}$, and let $(W, S)$ be the Coxeter system induced by the action. Then $W$ acts by reflections on $M_{1}$, and there is an $S$-panel submanifold fundamental domain $Q_{1}$ of $M_{1}$ such that $M_{1} \cong{ }_{W} \mathcal{U}\left(W, Q_{1}\right)$.

Proof. By Lemma 4.1. $W$ acts by reflections on $M_{1}$, and the set of elements of $W$ that act by reflections on $M_{1}$ is equal to the set of elements that act by reflections on $M_{2}$, which is equal to the set of reflections of $(W, S)$. Choose a fundamental domain $P$ of the $W$ action on $M_{1}$ and suppose that $T$ is the set of generating reflections determined by the action $t$. Then $M_{1} \cong{ }_{W} \mathcal{U}(W, P)$ ([13], Thm. 4.1). From now on, we will assume that $M_{1}$ is the universal space $\mathcal{U}(W, P)$. By [25], the manifold $M_{1}$ admits a $\operatorname{CAT}(0)$ locally flat metric, and $W$ acts on $M_{1}$ by isometries.

Since Coxeter manifolds are spaces of type $\mathcal{E} W$, any two are $W$-homotopy equivalent ([9]). Let $f: M_{1} \rightarrow M_{2}$ be a $W$-homotopy equivalence and $g$ a $W$-homotopy inverse of $f$. Since the action is properly discontinuous and cocompact, $f$ is a proper homotopy equivalence. Since $W$ acts by reflections on $M_{2}$, the generators $s \in S$ act as reflections on $M_{2}$ and there is an $S$-panel subspace $Q_{2}$ of $M_{2}$ such that $M_{2} \cong{ }_{W} \mathcal{U}\left(W, Q_{2}\right)$. The subspace $Q_{2}$ is the closure of a component of the non-singular set of $M_{2}$. Since $Q_{2}$ is also the orbit space of the action, it is compact.

For $R^{\prime} \subseteq R$ a set of reflections in $W$, set $M_{i}\left(R^{\prime}\right)=\bigcup_{r \in R^{\prime}} M_{i}^{s}, i=1,2$. In particular, $M_{i}(R)=\sigma\left(M_{i}\right)$.

Claim 1. $H_{n}\left(M_{2}, M_{2}(S)\right) \cong \mathbb{Z}$, and for each $s \in S, H_{n}\left(M_{2}, M_{2}(S-\{s\})\right)=0$.

Proof. The nerve of the cover of $M_{2}(S)$ by the walls $M_{2}^{s}(s \in S)$ is the same as the nerve of the cover of $\partial Q_{2}$ by the panels $Q_{2 s}$. Since all the intersections of the elements of the cover are contractible, the orbit map $p_{2}: M_{2} \rightarrow Q_{2}$ induces a homotopy equivalence $p_{2} \mid: M_{2}(S) \rightarrow \partial Q_{2}$ ([2], Thm. 8.2.2). Thus

$$
H_{n}\left(M_{2}, M_{2}(S)\right) \cong H_{n}\left(Q_{2}, \partial Q_{2}\right) \cong \mathbb{Z} \text {. }
$$

For the second part, set $S^{\prime}=S-\{s\}, W^{\prime}=W_{S^{\prime}}$. Then $W^{\prime}$ acts on $M_{2}$ by reflections. Let $Q_{2}^{\prime}$ be the closure of the component of the singular set of the $W^{\prime}$ action that contains $Q_{2}$. Then, for each $s^{\prime} \in S^{\prime}$,

$$
\operatorname{dim}\left(Q_{2}^{\prime} \cap M_{2 s^{\prime}}\right)=\operatorname{dim}\left(Q_{2} \cap M_{2 s^{\prime}}\right)=n-1 .
$$

Thus $Q_{2}^{\prime}$ is a fundamental domain of the $W^{\prime}$ action on $M_{2}$. By Lemma 4.4 $\operatorname{vcd}\left(W^{\prime}\right)<n$, and thus $Q_{2}^{\prime}$ is not compact, which implies

$$
H_{n}\left(M_{2}, M_{2}\left(S^{\prime}\right)\right) \cong H_{n}\left(Q_{2}^{\prime}, \partial Q_{2}^{\prime}\right)=0 .
$$


Claim 2. (i) $H_{n}\left(M_{1}, M_{1}(S)\right) \cong \mathbb{Z}$, and, for each $s \in S, H_{n}\left(M_{1}, M_{1}(S-\{s\})\right)=0$.

(ii) $M_{1}-M_{1}(S)$ contains a unique component with compact closure, but all the components of $M_{1}-M_{1}(S-\{s\})$ have non-compact closure.

Proof. The $W$-homotopy equivalence $f$ induces a homotopy equivalence of pairs

$$
f:\left(M_{1}, M_{1}\left(S^{\prime}\right)\right) \rightarrow\left(M_{2}, M_{2}\left(S^{\prime}\right)\right), \quad S^{\prime}=S \text { or } S-\{s\} .
$$

The result in (i) follows from Claim 1. For part (ii), notice that there is a sequence of isomorphisms $\left(S^{\prime}=S\right.$ or $\left.S^{\prime}=S-\{s\}\right)$

$$
\begin{aligned}
& \Gamma_{c}\left(M_{2}-M_{2}\left(S^{\prime}\right)\right) \stackrel{J_{2}^{-1}}{\longrightarrow} H_{n}\left(M_{2}, M_{2}\left(S^{\prime}\right)\right) \stackrel{f_{*}}{\longrightarrow} H_{n}\left(M_{1}, M_{1}\left(S^{\prime}\right)\right) \\
& \stackrel{J_{1}}{\longrightarrow} \Gamma_{c}\left(M_{1}-M_{1}\left(S^{\prime}\right)\right),
\end{aligned}
$$

where $\Gamma_{c}(-)$ denotes the free abelian group on the components of the space which have compact closure ([19], Ch. VIII, Proposition 3.3). The result follows from part (i).

Claim 3. Let $Q_{1}$ be the closure of the unique component of $M_{1}-M_{1}(S)$ with compact closure. Then:

(i) $Q_{1}$ is homeomorphic to a compact manifold with boundary, and it is a totally geodesic subspace of $M_{1}$.

(ii) $\operatorname{dim}\left(Q_{1} \cap M_{1}^{s}\right)=n-1$, for all $s \in S$.

(iii) The space $Q_{1}$ is a union of an odd number of domains, i.e.

$$
Q_{1}=\bigcup_{j=1}^{2 k+1} w_{j} P
$$

(iv) Set

$$
\left[Q_{1}\right]^{*}=\sum_{j=1}^{2 k+1}\left[w_{j} P\right]^{*} \in H_{c}^{n}\left(M_{1}, \sigma\left(M_{1}\right)\right)
$$

$\left(\mathbb{Z} / 2 \mathbb{Z}\right.$-coefficients are understood). Then $f^{*}\left(\left[Q_{2}\right]^{*}\right)=\left[Q_{1}\right]^{*}$.

Proof. (i) For each $s \in S$, the fixed point set $M_{1}^{s}$ is a totally geodesic subspace of $M_{1}$. Furthermore, $M_{1}-M_{1}^{s}=\operatorname{int}\left(M_{1}^{s}(+)\right) \cup \operatorname{int}\left(M_{1}^{s}(-)\right)$ as a union of two components, and the closure of each component is a totally geodesic subspace of $M_{1}$. The space $Q_{1}$ is the intersection of the half spaces that contain it, i.e. it is the intersection of totally geodesic subspaces and thus it is totally geodesic. Since $s \in S$ acts by reflections on $M_{1}, Q_{1}$ is a manifold with boundary.

We prove (ii) by contradiction. Let $s \in S$ be such that $\operatorname{dim}\left(Q_{1} \cap M_{1}^{s}\right)<n-1$. Then the set $M_{1}-M_{1}(S-\{s\})$ has a component with compact closure $Q_{1}$, contradicting Claim 2(ii).

For (iii), we use homology and cohomology with $\mathbb{Z} / 2 \mathbb{Z}$-coefficients. The group $H_{n}\left(M_{i}, M_{i}(S)\right)$ is generated by the fundamental class of $\left(Q_{i}, \partial Q_{i}\right)$, for $i=1,2$. Since

$$
f_{*}: H_{n}\left(M_{1}, \sigma\left(M_{1}\right)\right) \rightarrow H_{n}\left(M_{2}, \sigma\left(M_{2}\right)\right)
$$

is an isomorphism of free $\mathbb{Z} / 2 \mathbb{Z}[W]$-modules in one generator (Lemma 4.6), there is a unit $u$ in $\mathbb{Z} / 2 \mathbb{Z}[W]$ such that $f_{*}([P])=u\left(\left[Q_{2}\right]\right)$. The element $u$ is the sum of an odd number of elements of the group ring, because $u$ is mapped to the unit of $\mathbb{Z} / 2 \mathbb{Z}$ under the augmentation map. The same is true for the inverse $u^{-1}$. Now let

$$
f_{S *}: H_{n}\left(M_{1}, M_{1}(S)\right) \rightarrow H_{n}\left(M_{2}, M_{2}(S)\right)
$$


and each group is a $\mathbb{Z} / 2 \mathbb{Z}$-vector space of dimension 1 . Thus $f_{S *}\left(\left[Q_{1}, \partial Q_{1}\right]\right)=$ $\left[Q_{2}, \partial Q_{2}\right]$. By the commutativity of the diagram

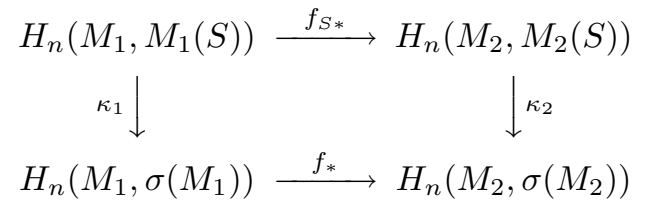

we derive that

$$
\begin{aligned}
{\left[Q_{2}\right] } & =\kappa_{2} \circ f_{S *}\left(\left[Q_{1}\right]\right)=f_{*} \circ \kappa_{1}\left(\left[Q_{1}\right]\right) \\
& \Rightarrow \kappa_{1}\left(\left[Q_{1}\right]\right)=\left(f_{*}\right)^{-1}\left(\left[Q_{2}\right]\right)=u^{-1}[P] \\
& \Rightarrow \kappa_{1}\left(\left[Q_{1}\right]\right)=\sum_{j=1}^{2 k+1}\left[w_{j} P\right] .
\end{aligned}
$$

By the definition of the map $\kappa_{1}$,

$$
Q_{1}=\bigcup_{j=1}^{2 k+1} w_{j} P
$$

Part (iv) follows from the fact that $f^{*}$ is the $\mathbb{Z} / 2 \mathbb{Z}[W]$-dual of the map $f_{*}$ (Lemma 4.6).

Claim 4. For each $R^{\prime} \subset R$, the natural set of generators of $H_{c}^{n}\left(M_{i}, M_{i}\left(R^{\prime}\right)\right)$ is the set corresponding to the components of $M_{i}-M_{i}\left(R^{\prime}\right)$ under the Poincaré duality isomorphism $(i=1,2)$. Then for each $r \in R$, the inclusion induced map

$$
\iota_{i r}: H_{c}^{n}\left(M_{i}, \sigma\left(M_{i}\right)\right) \rightarrow H_{c}^{n}\left(M_{i}, M_{i}^{r}\right), \quad i=1,2,
$$

maps $\left[w Q_{1}\right]^{*}$ to a natural generator. Furthermore, the diagram commutes:

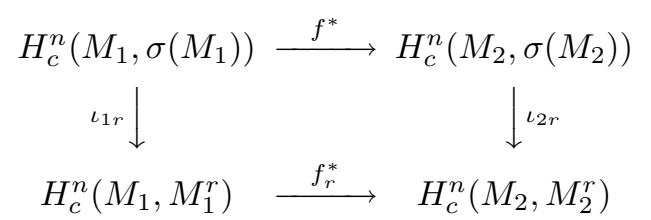

In particular, if $M_{i}^{r}(+)$ demotes the closure of the component of $M_{i}-M_{i}^{r}$ such that $\iota_{i r}\left(\left[Q_{i}\right]^{*}\right)=\left[M_{i}^{r}(+)\right]^{*}(i=1,2, r \in R)$, then $f_{r}^{*}\left(\left[M_{1}^{r}(+)\right]^{*}\right)=\left[M_{2}^{r}(+)\right]^{*}$.

Proof. By Claim 3, we know that

$$
w Q_{1}=\bigcup_{j=1}^{2 k+1} w w_{j} P
$$

Let $M_{1}-M_{1}^{r}=M_{1}(+) \cup M_{1}^{r}(-)$, the union of two closed half-spaces whose intersection in $M^{r}$. We consider two cases

Case 1. Let $w Q_{1}$ be contained in the closure of one of the half-spaces determined by $r$, say $M_{1}^{r}(+)$. Then $w w_{j} P$ is contained in $M_{1}^{r}(+)$ for $j=1, \ldots, 2 k+1$. If $\left[M_{1}^{r}(+)\right]^{*}$ denotes the natural generator of $H_{c}^{n}\left(M_{1}, M_{1}^{r}\right)$ represented by $M_{1}^{r}(+)$, then

$$
\iota_{1 r}\left(\left[w w_{j} P\right]^{*}\right)=\left[M_{1}^{r}(+)\right]^{*} \Rightarrow \iota_{1 r}\left(\left[w Q_{1}\right]^{*}\right)=\sum_{j=1}^{2 k+1} \iota_{1 r}\left(\left[w w_{j} P\right]^{*}\right)=\left[M_{1}^{r}(+)\right]^{*} .
$$

Case 2. If $w Q_{1}$ is not contained in any of $M_{1}^{r}( \pm)$, then $M_{1}^{r} \cap \operatorname{int}\left(w Q_{1}\right) \neq \emptyset$. Since $w Q_{1}, M_{1}^{r}( \pm)$ are totally geodesic subspaces of $M_{1}$, the intersections $w Q_{1} \cap M_{1}^{r}( \pm)$ are totally geodesic and therefore connected. Each intersection is a union of chambers. Since the number of chambers in $w Q_{1}$ is odd, one intersection will contain 
an odd number of chambers and the other an even number. Then, $\iota_{1 r}\left(\left[w Q_{1}\right]^{*}\right)$ is equal to the generator corresponding to the half-space containing an odd number of chambers.

The last assertion of the claim follows from Lemma 4.5.

Claim 5. For each $r \in R$, define

$$
P_{r}^{\prime}=\left\{w \in W: \iota_{1 r}\left(\left[w Q_{1}\right]^{*}\right)=\iota_{1 r}\left(\left[Q_{1}\right]^{*}\right)\right\} .
$$

Then $P_{r}^{\prime}=P_{r}=\{w \in W: \ell(r w)>\ell(w)\}$, where the length is relative to the generating set $S$.

Proof. Claim 4 implies that the element $\iota_{1 r}\left(\left[w Q_{1}\right]^{*}\right)$ is a natural generator, for all $w \in W$. Since the action of $W$ on $M_{2}$ is by reflections with fundamental domain $Q_{2}, P_{r}$ is the set of all $w \in W$ such that $w Q_{2}$ and $Q_{2}$ belong to the same half-space bounded by $M^{r}\left([13]\right.$, Remark 7.4) or equivalently, $\iota_{2 r}\left(\left[w Q_{2}\right]^{*}\right)=\iota_{2 r}\left(\left[Q_{2}\right]^{*}\right)$. Since the diagram

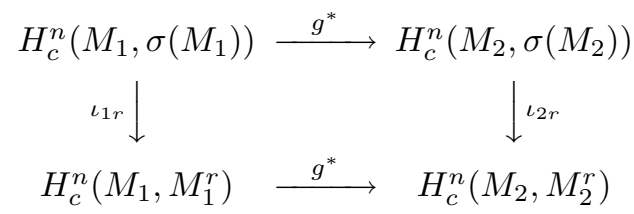

commutes, we have the following:

$$
\begin{aligned}
w \in P_{r}^{\prime} & \Leftrightarrow \iota_{1 r}\left(\left[w Q_{1}\right]^{*}\right)=\iota_{1 r}\left(\left[Q_{1}\right]\right)^{*} \\
& \Leftrightarrow g^{*} \iota_{1 r}\left(\left[w Q_{1}\right]^{*}\right)=g^{*} \iota_{1 r}\left(\left[Q_{1}\right]^{*}\right) \\
& \Leftrightarrow \iota_{2 r} g^{*}\left(\left[w Q_{1}\right]^{*}\right)=\iota_{2 r} g^{*}\left(\left[Q_{1}\right]^{*}\right) \\
& \Leftrightarrow \iota_{2 r}\left(\left[w Q_{2}\right]^{*}\right)=\iota_{2 r}\left(\left[Q_{2}\right]^{*}\right)
\end{aligned}
$$

(the last implication is a consequence of Claim 3(iv) and the fact that $g^{*}$ is $W$ equivariant), which means that $w Q_{2}$ and $Q_{2}$ are on the same side of $M_{2}^{r}$.

Claim 6. Let $s, s^{\prime} \in S, s \neq s^{\prime}$. Then $M_{1}^{s}(+) \cap M_{1}^{s^{\prime}}(+)$ is a fundamental domain of the action of the dihedral group $\left\langle s, s^{\prime}\right\rangle$.

Proof. Let $r$ be a reflection in $\left\langle s, s^{\prime}\right\rangle$ of shortest length (in the generators $s$ and $\left.s^{\prime}\right)$ such that $M_{1}^{r} \cap\left(M_{1}^{s}(+) \cap M_{1}^{s^{\prime}}(+)\right) \neq \emptyset$. We can assume that the order of $s s^{\prime}$ is greater than two, because if it is equal to 2, there are no reflections in $\left\langle s, s^{\prime}\right\rangle$ other than $s$ and $s^{\prime}$. Without loss of generality we can assume that $r=s^{\prime} r^{\prime} s^{\prime}$ is a reduced expression of $r$. We will give the proof in the case that the dihedral group generated by $s$ and $s^{\prime}$ is finite. The other case follows similarly. The space $M_{1}^{r}$ separates $M_{1}^{s} \cap\left(M_{1}^{s}(+) \cap M_{1}^{s^{\prime}}(+)\right)$ and $M_{1}^{s^{\prime}} \cap\left(M_{1}^{s}(+) \cap M_{1}^{s^{\prime}}(+)\right)$. Since $Q_{1}$ intersects both intersections in a codimension 1 submanifold, $M_{1}^{r} \cap \operatorname{int}\left(Q_{1}\right) \neq \emptyset$. Let

$$
\iota_{i}: H_{c}^{n}\left(M_{i}, \sigma\left(M_{i}\right)\right) \rightarrow H_{c}^{n}\left(M_{i}, M_{i}\left(\left\{s, s^{\prime}, r\right\}\right)\right), \quad i=1,2,
$$

be the inclusion induced maps. The following diagram commutes:

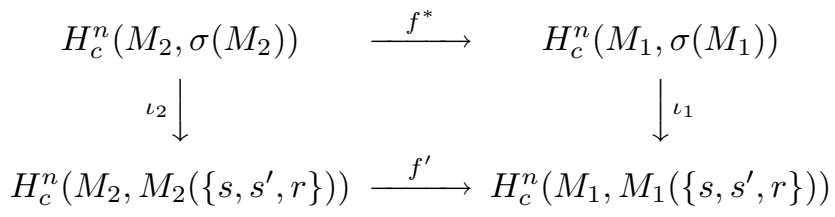




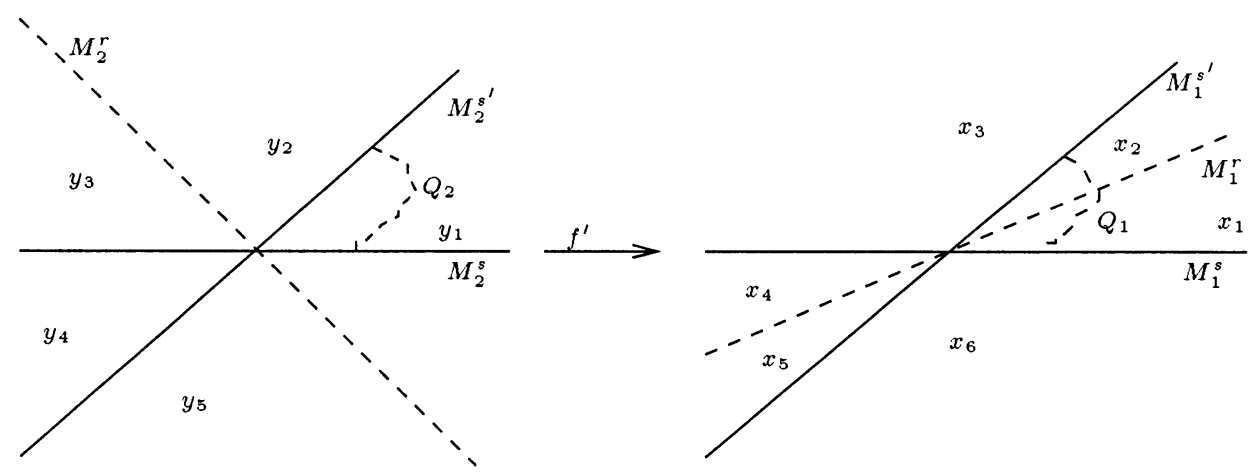

FiguRE 1. Schematic representation of the regions deteremined by the three walls $M_{i}^{s}, M_{i}^{s^{\prime}}, M_{i}^{r}$.

(where $f^{\prime}$ is induced by $f$ ) and the two horizontal maps are isomorphisms. We represent the generators of the groups $H_{c}^{n}\left(M_{i}, M_{i}\left(\left\{s, s^{\prime}, r\right\}\right)\right), i=1,2$, schematically in Figure 1.

If $s^{\prime}\left(\operatorname{int}\left(Q_{1}\right)\right) \cap M_{1}^{r} \neq \emptyset$, then $\operatorname{int}\left(Q_{1}\right) \cap M_{1}^{r^{\prime}} \neq \emptyset$, contradicting the minimality of the length of $r$. Thus $s^{\prime}\left(\operatorname{int}\left(Q_{1}\right)\right) \cap M_{1}^{r}=\emptyset$ and $s^{\prime} Q_{1} \subset x_{3} \cup x_{4}$. Since $s^{\prime} Q_{1}$ is the union of an odd number of domains and $M_{1}^{s}$ separates it into two components, either $x_{3}$ or $x_{4}$ contains an odd number of components. Then $\iota_{1}\left(\left[s^{\prime} Q_{1}\right]^{*}\right)$ is equal to either $x_{3}$ or $x_{4}$. If $\iota_{1}\left(\left[s^{\prime} Q_{1}\right]^{*}\right)=x_{4}$, then $\iota_{1 s}\left(\left[s^{\prime} Q_{1}\right]^{*}\right)=\left[M_{1}^{s}(-)\right]^{*}$, contradicting Claim 5 . Thus $\iota_{1}\left(\left[s^{\prime} Q_{1}\right]^{*}\right)=x_{3}$, which implies that $f^{\prime}\left(y_{2}\right)=x_{3}$. Also, $r s^{\prime} Q_{1} \subset x_{1} \cup x_{5} \cup x_{6}$, which implies that $\iota_{1}\left(\left[r s^{\prime} Q_{1}\right]^{*}\right)=a_{1} x_{1}+a_{5} x_{5}+a_{6} x_{6}$.

If $\ell\left(s r s^{\prime}\right)<\ell\left(r s^{\prime}\right)$, then $r s^{\prime}=s r^{\prime \prime}$, which cannot happen in a dihedral group for a reflection $r$. Thus $\ell\left(s r s^{\prime}\right)>\ell\left(r s^{\prime}\right)$. In particular, $\iota_{2}\left(\left[r s^{\prime} Q_{2}\right]^{*}\right)=y_{3}$ (because $r s^{\prime} Q_{2}$ must lie on the opposite side of $M_{2}^{s^{\prime}}$ and $M_{2}^{r}$, and on the same side of $M_{2}^{s}$ as $\left.Q_{2}\right)$. Then

$$
\begin{aligned}
f^{\prime}\left(y_{3}\right) & =f^{\prime}\left(\iota_{2}\left(\left[r s^{\prime} Q_{2}\right]^{*}\right)\right) \\
& =\iota_{1}\left(f^{*}\left(\left[r s^{\prime} Q_{2}\right]^{*}\right)\right) \\
& =\iota_{1}\left(\left[r s^{\prime} Q_{1}\right]^{*}\right) \\
& =a_{1} x_{1}+a_{5} x_{5}+a_{6} x_{6}
\end{aligned}
$$

Let

$$
j_{i}: H_{c}^{n}\left(M_{i}, M_{i}\left(\left\{s, s^{\prime}, r\right\}\right)\right) \rightarrow H_{c}^{n}\left(M_{i}, M_{i}^{s} \cup M_{i}^{s^{\prime}}\right)
$$

be the inclusion induced map $(i=1,2)$. Then the following diagram commutes:

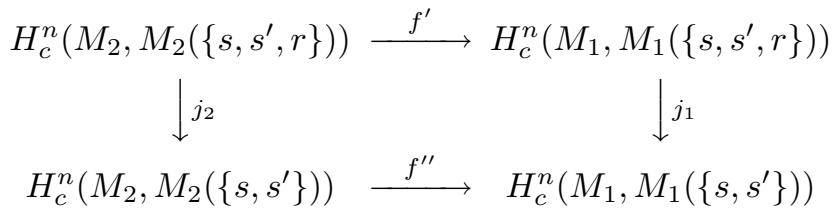


Using the fact that $j_{2}\left(y_{2}\right)=j_{2}\left(y_{3}\right)$, we have

$$
\begin{aligned}
& f^{\prime \prime}\left(j_{2}\left(y_{2}\right)\right)=f^{\prime \prime}\left(j_{2}\left(y_{3}\right)\right) \\
& \quad \Rightarrow j_{1}\left(f^{\prime}\left(y_{2}\right)\right)=j_{1}\left(f^{\prime}\left(y_{3}\right)\right) \\
& \quad \Rightarrow j_{1}\left(x_{3}\right)=j_{1}\left(a_{1} x_{1}+a_{5} x_{5}+a_{6} x_{6}\right)
\end{aligned}
$$

which cannot occur. Contradiction.

For the case of the infinite dihedral group, the same method works. In this case, the proof actually is outlined in [33, Proposition 4, part (ii).

Claim 7 . The space $Q_{1}$ is a fundamental domain of the $W$-action on $M_{1}$.

Proof. The proof of the Claim 7 is classical. Claim 6 implies that $M_{1}^{s}(+) \cap M_{1}^{s^{\prime}}(+)$ is the fundamental domain of the action of the dihedral group generated by $s$ and $s^{\prime}$. Thus, for all $w$ in the dihedral group,

(i) $w\left(M_{1}^{s}(+) \cap M_{1}^{s^{\prime}}(+)\right) \subset M_{1}^{s}(+)$ and $\ell(s w)=\ell(w)+1$, or

(ii) $w\left(M_{1}^{s}(+) \cap M_{1}^{s^{\prime}}(+)\right) \subset s M_{1}^{s}(+)$ and $\ell(s w)=\ell(w)-1$.

The rest of the proof follows as in [23], Theorem 2.1 (also 32, [33]).

Thus $Q_{1}$ is the closure of a component of the non-singular set. Since $\operatorname{dim}\left(Q_{1} \cap M_{1}^{s}\right)=n-1$ (for all $s \in S$ ), it contains points which are fixed only by $s$. By definition $Q_{1}$ is an $S$-panel fundamental domain for the $W$-action on $M_{1}$.

Combining Proposition 3.6 and Proposition 4.7, we have a uniqueness statement on the manifold-reflection presentation of a Coxeter group.

Theorem 4.8. Let $(W, T)$ and $(W, S)$ be two manifold-reflection systems in $W$. Then there is an inner automorphism of $W$ that maps $T$ to $S$, i.e., $T$ and $S$ are conjugate.

Proof. By Theorem 15.3 in 13, there are two contractible manifolds $M_{T}$ and $M_{S}$ such that $W$ acts cocompactly by reflections on both of them, and the boundary of the fundamental domain is $\left|K_{0}(W, T)\right|$ in the first case and $\left|K_{0}(W, S)\right|$ in the second (as panel manifolds). The manifolds $M_{T}$ and $M_{S}$ are both spaces of type $\mathcal{E} W$ by Proposition 3.6. Thus they are Coxeter manifolds. Write $C$ for the chamber in $M_{T}$ with boundary panel homeomorphic to $\left|K_{0}(W, T)\right|$. By Proposition $4.7 M_{T}$ admits a fundamental domain $C^{\prime}$, with boundary panel homeomorphic to $\left|K_{0}(W, S)\right|$. Thus $M_{T}$ admits two chambers, one determining the Coxeter generators $T$ and the other the Coxeter generators $S$. By Theorem 4.1(iii) in [13, there is an element $w \in W$ that maps one chamber to the other. Then conjugation by $w$ induces a bijection between $S$ and $T$.

Remark 4.3. In a recent preprint ([8]), R. Churney and M. Davis have proved the result in Theorem 4.8 using more combinatorial methods. Actually their methods produce a more general result. More specifically, they derive the same uniqueness result by assuming that the Coxeter group admits an action by reflections on certain pseudo-manifolds that are not necessarily manifolds.

\section{Structure Set Rigidity Theorems}

In this section we are going to prove the rigidity theorems for the structure set of manifolds equipped with a Coxeter group action, not necessarily by reflections. 
Let $M$ be a Coxeter manifold without boundary. Since $W$ acts properly discontinuously and cocompactly on $M, W$ is a virtual Poincaré duality group. By Theorem B in [16], $(W, S) \cong\left(W_{1}, S_{1}\right) \times\left(W_{2}, S_{2}\right)$, where $W_{2}$ is a finite Coxeter group and $\left(W_{1}, S_{1}\right)$ is a manifold-reflection system. Notice that $M$ is a Coxeter $W_{1}$-manifold. By Lemma 4.2 the group $W_{2}$ acts trivially on $M$.

We need the following lemma from surgery theory, which is derived using the Poincaré conjecture ([26], Lemma 3.1; 35]; [20], for $n=4)$.

Lemma 5.1. Let $\left(M^{n}, \partial M\right)$ be a compact contractible manifold with boundary. Then the relative structure set

$$
\mathcal{S}\left(M^{n}, \partial M\right)=* \text { for } n \neq 3 .
$$

The next lemma will be used in the construction of homeomorphisms of faces of dimension 3 .

Lemma 5.2. Let $(M, \partial M)$ be a compact three dimensional contractible manifold embedded into $S^{3}$. Then $(M, \partial M)$ is homeomorphic to the pair $\left(D^{3}, S^{2}\right)$.

Proof. The boundary of $M$ is a surface of genus zero. Therefore $\partial M$ is homeomorphic to $S^{2}$. By the Schoenflies lemma ([7]), $S^{3}-S^{2}$ consists of two components whose closures are homeomorphic to the 3 -disc. Therefore $M$ is homeomorphic to a 3-disc.

We can now prove the structure set rigidity theorem for Coxeter groups. As always, $W$ will be a Coxeter group. The setting will be the following:

Let $M^{n}$ be a Coxeter $W$-manifold without boundary. Let $N^{n+k}$ be a Coxeter $W$-manifold, and let

$$
f:(N, \partial N) \rightarrow\left(M \times D^{k}, M \times S^{k-1}\right)
$$

be a $W$-map such that $f \mid \partial N$ is a $W$-homeomorphism (the $W$-action on $D^{k}$ is trivial).

We need the following assumption on the three dimensional fixed point sets

Definition 5.1. Let $\Gamma$ be a discrete group acting on a manifold $X$. The pair $(\Gamma, X)$ satisfies the dimension three condition if the three dimensional fixed point sets of $X$ can be embedded into $S^{3}$.

Theorem 5.3. Let $(W, S)$ be a Coxeter system and $f$ a $W$-map as above. Assume that $(W, N)$ and $\left(W, M \times D^{k}\right)$ satisfy the dimension three condition. Then $f$ is $W$-homotopic to a $W$-homeomorphism.

Proof. By assumption both spaces $N$ and $M \times D^{k}$ are spaces of type $\mathcal{E} W$. Thus $f$ is a $W$-homotopy equivalence. Since $M$ is a Coxeter manifold, $W$ is a virtual Poincaré duality group. Thus there is a decomposition $(W, S) \cong\left(W_{1}, S_{1}\right) \times\left(W_{2}, S_{2}\right)$ with $\left(W_{1}, S_{1}\right)$ a manifold-reflection system and $W_{2}$ a finite Coxeter group. By Lemma 4.2, $W_{2}$ acts trivially on $M$. By Lemma 4.1)(1), $W_{1}$ acts by reflections on $M$, and by Theorem 4.8 there is an $S$-panel manifold which is the fundamental domain of the $W_{1}$-action on $M$, which is admissible (Proposition 3.6). Since the action of $W_{1}$ on $D^{k}$ is trivial, $W_{1}$ acts by reflections on $M \times D^{k}$ as well as on $M \times S^{k-1}$. Then $Q_{1}=Q \times D^{k}$ is a fundamental domain of the $W_{1}$-action on $M \times D^{k}$, which is also admissible. 
By Lemma 4.2, $W_{2}$ acts trivially on $N$. Therefore it is enough to show that $f$ is $W_{1}$-homotopic to a homeomorphism. For the rest of the proof we assume that $(W, S)$ is a manifold-reflection system.

By Lemma 4.T 2), $W$ acts by reflections on $N$. Let $Q_{2}$ be the $T$-panel fundamental domain determined by the action and $N \cong_{W} \mathcal{U}\left(W, Q_{2}\right)$.

Claim 1. $(W, T)$ is a manifold-reflection system.

Proof. By Theorem B in [16], $(W, T)$ splits as the product of $\left(W_{1}, T_{1}\right)$ and $\left(W_{2}, T_{2}\right)$, where $\left(W_{1}, T_{1}\right)$ is a manifold-reflection system and $\left(W_{2}, T_{2}\right)$ is finite. By Lemma 4.2 $W_{2}$ acts trivially on $N$. Unless $W_{2}$ is the trivial group, this is impossible because $W$ acts on $N$ by reflections. Thus $(W, T)$ is a manifold-reflection system.

By Theorem 4.8, $T$ and $S$ are conjugate in $W$. Thus, by choosing another chamber in $N$, we can assume that $Q_{2}$ is an $S$-panel space and it is admissible by Proposition 3.6.

Since there are $W$-homeomorphisms

$$
M \times D^{k} \cong{ }_{W} \mathcal{U}\left(W, Q_{1}\right), \quad N \cong{ }_{W} \mathcal{U}\left(W, Q_{2}\right),
$$

$f$ induces a $W$-homotopy equivalence:

$$
g: \mathcal{U}\left(W, Q_{2}\right) \rightarrow \mathcal{U}\left(W, Q_{1}\right)
$$

We will construct an $S$-panel homeomorphism $\lambda: Q_{2} \rightarrow Q_{1}$. The construction is done inductively on the faces. We first show how to extend the function to three dimensional panels.

Claim 2. Let $Q_{i L}(i=1,2)$ be 3 -dimensional faces and

$$
\lambda:\left(Q_{2 L}, \partial Q_{2 L}\right) \rightarrow\left(Q_{1 L}, \partial Q_{1 L}\right)
$$

an $S$-panel homotopy equivalence such that $\lambda \mid \partial Q_{2 L}$ is a homeomorphism. Then $\lambda$ is homotopic to a homeomorphism relative to $\partial Q_{2 L}$.

Proof. By the definition of faces, $Q_{i L}$ is contained in a fixed-point set of dimension $3(i=1,2)$. Since the pairs $\left(W, M \times D^{k}\right)$ and $(W, N)$ satisfy the dimension three condition, $Q_{i L}$ is a compact contractible 3 -manifold in $S^{3}$. Lemma 5.2 implies that $\left(Q_{i L}, \partial Q_{2 L}\right)$ is homeomorphic to $\left(D^{3}, S^{2}\right)$. Since every homeomorphism of $S^{2}$ extends to a homeomorphism on $D^{3}, \lambda \mid \partial Q_{2 L}$ extends to a homeomorphism $\lambda$ on $Q_{2 L}$ which is homotopic to $\lambda$.

Now we start the induction argument. Let $Q_{i K}, i=1,2$, be faces of lowest dimension. Then there is a finite subgroup $H$ of $W$ such that

$$
Q_{1 K}=M^{H} \times D^{k}, \quad Q_{2 K}=N^{H} .
$$

The subgroup $H$ is a maximal isotropy subgroup of the $W$-action. If $k=0$, then $Q_{i K}(i=1,2)$ is a contractible manifold without boundary; thus $Q_{i K}$ is a point. Therefore, $Q_{1 K}$ and $Q_{2 K}$ are homeomorphic. If $k>0$, then $Q_{i K}(i=1,2)$ is a contractible manifold with boundary. The boundaries are

$$
\partial Q_{1 K}=M^{H} \times S^{k-1}, \quad \partial Q_{2 K}=(\partial N)^{H} .
$$

But $f \mid \partial Q_{2 K}$ is a homeomorphism by assumption. Since the pair $\left(Q_{2 K}, \partial Q_{2 K}\right)$ satisfies the homotopy extension property and $Q_{1 K}$ is contractible, we can extend $f \mid \partial Q_{2 K}$ to a homotopy equivalence $\lambda_{K}$ on $Q_{2 K}$. By Lemma 5.1] $\lambda_{K}$ is homotopic (relative to the boundary) to a homeomorphism (we use Claim 2 if the dimension is equal to three). 
In general, the boundary of a face $Q_{2 J}$ is $\delta Q_{2 J} \cup\left(Q_{2 J} \cap \partial N\right)$. By induction the homeomorphism $\lambda_{\partial Q_{2 J}}$ has been defined on $\delta Q_{2 J}$ (which is a union of panels of smaller dimension) and we can set $\lambda$ to be $f$ when restricted to $Q_{2 J} \cap \partial N$. We extend $\lambda_{\partial Q_{2 J}}$ to a homotopy equivalence $\lambda_{Q_{2 J}}^{\prime}$ on $Q_{2 J}$. By Lemma 5.1 (or by Claim 2 if the dimension of $Q_{2 J}$ is 3) $\lambda_{Q_{2 J}}^{\prime}$ is homotopic to a homeomorphism $\lambda_{Q_{2 J}}$. By repeating the construction, we get an $S$-panel homeomorphism $\lambda: Q_{2} \rightarrow Q_{1}$. Then $\mathcal{U}(W, \lambda) \simeq_{W} g([9)$ and $\mathcal{U}(W, \lambda)$ is a $W$-homeomorphism (Section 2). Therefore

$$
f \simeq_{W} g \simeq_{W} \mathcal{U}(W, \lambda)
$$

The last map is a $W$-homeomorphism.

\section{K-THEORY Rigidity ThEOREMS}

We will extend the constructions developed in Section 5 to show rigidity of equivariant $h$-cobordisms induced by Coxeter groups acting on contractible manifolds. More precisely, let $W$ be a Coxeter group and $M$ a Coxeter $W$-manifold without boundary. Let $W_{0}$ be a normal torsion free subgroup of finite index, and let $G=W / W_{0}$. Then the manifold $M_{0}=M / W_{0}$ is a closed $G$-manifold. We will show that the Whitehead group that classifies isovariant $G$-h-cobordisms over $M_{0} \times T^{n}$ vanishes, i.e., $W h_{G}^{T o p, \rho}\left(M_{0} \times T^{n}\right)=0([28],[29])$.

Let $M$ be a Coxeter $W$-manifold without boundary, and $T^{n}$ the $n$-dimensional torus with the trivial $W$-action. Then $(W, S)=\left(W_{1}, S_{1}\right) \times\left(W_{2}, S_{2}\right)$, where $\left(W_{1}, S_{1}\right)$ is a manifold-reflection system and $W_{2}$ is a finite Coxeter group. The subgroup $W_{1}$ acts on $M$ by reflections (Lemma 4.1). The fundamental domain $Q$ of the action is an admissible $S$-panel manifold. Thus $W_{1}$ acts by reflections on $M \times T^{n}$ with fundamental domain $Q \times T^{n}$.

Definition 6.1. An $S$-panel h-cobordism $\left(X ; Q_{1}, Q_{2}\right)$ consists of a $W$-finite $S$ panel manifold $\left(X,\left(X_{s}\right)_{s \in S}\right)$ such that $\left(X_{J} ; Q_{1 J}, Q_{2 J}\right)$ is an $h$-cobordism, for each $J \in \mathcal{F}(W, S)$. An $S$-h-cobordism $X$ is called trivial if $X$ is $S$-panel homeomorphic to $Q_{1} \times I$ with the natural product panel structure.

Lemma 6.1. Let $\tau=\left(X ; Q_{1} \times T^{n}, Q_{2}\right)$ be an $S$-panel cobordism which is trivial when restricted to faces of dimension less than 4. If $Q_{1}$ is an admissible panel manifold, then $\tau$ is trivial.

Proof. Inductively, we extend the trivialization over each face, as in the proof of Theorem 5.3 (in dimension 4 we use Theorem 7.1A in [21]). The first step of the induction works because $W h\left(\pi_{1}\left(T^{n}\right)\right)=0$.

The next lemma is the analogue of Theorem 4.8 for $W$-isovariant maps. The proof is easier in this case.

Lemma 6.2. Let $M_{i}^{n_{i}}, i=1,2$, be two $W$-manifolds on which $W$ acts properly discontinuously and cocompactly. Assume that $W$ acts on $M_{2}$ by reflections and $(W, S)$ is a Coxeter system induced on $W$ by the action. We also assume that $M_{1}^{s}$ is a codimension 1 submanifold of $M_{1}$, for each $s \in S$. Let $f: M_{1} \rightarrow M_{2}$ be an isovariant homotopy equivalence. Then there is an $S$-panel manifold $Q_{1}$ such that $M_{1} \cong{ }_{W} \mathcal{U}\left(W, Q_{1}\right)$.

Proof. The isovariance of $f$ implies that $W$ acts by reflections on $M_{1}$. Let $g$ be an isovariant homotopy inverse of $f$. Since $g$ is isovariant, it induces a homotopy 
equivalence:

$$
g \mid: M_{2}-\bigcup_{r \in R} M_{2}^{r} \rightarrow M_{1}-\bigcup_{r \in R} M_{1}^{r},
$$

where $R$ is the set of reflections in $(W, S)$. Let $Q_{1}$ be the closure of the component corresponding to $Q_{2}$. For $s \in S, Q_{2}$ contains elements fixed only by $s$. Since $g$ is isovariant, $Q_{1}$ contains elements fixed only by $s$. Thus $Q_{1}$ is an $S$-panel fundamental domain of $M_{1}$.

Let $h=\left(N_{0} ; M_{0} \times T^{n}, M_{0}^{\prime}\right)$ be an isovariant $G$ - $h$-cobordism representing an element in $W h_{G}^{T o p, \rho}\left(M_{0} \times T^{n}\right)([28])$. Then $h$ is an isovariant $G$ - $h$-cobordism. We assume that $h$ is a trivial $h$-cobordism on fixed point sets of dimension less than 4 . The universal cover of $h$ is a $W$-h-cobordism $\tilde{h}=\left(N ; M \times T^{n}, M^{\prime}\right)$. By Lemma 4.2. the finite subgroup $W_{2}$ of $W$ acts trivially on $M$ and thus on all the spaces involved. So we can assume as before that $(W, S)$ is a manifold-reflection system. Since $M^{\prime}$ is isovariantly $W$-homotopy equivalent to $M \times T^{n}$, and $W$ acts by reflections on $M \times T^{n}, W$ acts by reflections on $M^{\prime}$. Let $Q^{\prime}$ be the $S$-panel manifold which is the fundamental domain of the action (Lemma 6.2).

Lemma 6.3. The $W$-cobordism $\tilde{h}$ is $W$-trivial.

Proof. Let $s \in S$. The triple $\left(N^{s}, M^{s} \times T^{n}, M^{\prime s}\right)$ is an $h$-cobordism, and thus $N^{s}$ is a codimension 1 submanifold of $N, W$-isovariantly homotopic to $M \times T^{n}$. By Lemma 6.2. $W$ acts by reflections on $N$ and there is an $S$-panel fundamental domain $X$ of the action. Then $\tau=\left(X ; Q \times T^{n}, Q^{\prime}\right)$ is an $S$-panel $h$-cobordism which satisfies the assumptions of Lemma 6.1 Thus $\tau$ is trivial, i.e. $X$ is $S$-panel homeomorphic to $Q \times T^{n}$. Therefore

$$
N \cong_{W} \mathcal{U}(W, X) \cong_{W} \mathcal{U}\left(W, Q \times T^{n} \times I\right) \cong_{W} \mathcal{U}(W, Q) \times T^{n} \times I \cong_{W} M \times T^{n} \times I .
$$

and the cobordism $\tilde{h}$ is $W$-trivial.

Theorem 6.4. If $M_{0}$ and $G$ are as in the beginning of the section, then

$$
W h_{G}^{T o p, \rho}\left(M_{0} \times T^{n}\right)=0 .
$$

Proof. Let $h=\left(N_{0} ; M_{0}, M_{0}^{\prime}\right)$ be a $G$-h-cobordism representing an element in the Whitehead group. By Lemma 6.3, the universal cover of $h, \tilde{h}=\left(N ; M \times T^{n}, M^{\prime}\right)$, is $W$-trivial, i.e. $N \cong{ }_{W} M \times T^{n} \times I$. Taking the orbit spaces under the $W_{0}$ action, we conclude that $h$ is trivial.

\section{REFERENCES}

1. M. Bestvina, The virtual cohomological dimension of Coxeter groups', Geometric Group Theory Vol. 1, LMS Lecture Notes 181, 19-23. MR 94g:20056

2. A. Borel and J-P. Serre, Corners and arithmetic groups, Comment. Math. Helv. 48, 436 - 491 (1973). MR 52:8337

3. N. Bourbaki, Groupes et algèbres de Lie, Ch. 4-6, Hermann, Paris, 1968. MR 39:1590

4. B. Brink and R. B. Howlett, A finiteness property and an automatic structure for Coxeter groups, Math. Ann. 296179 - 190 (1993). MR 94d:20045

5. K. S. Brown, Buildings, Springer-Verlag, New York, 1989. MR 90e:20001

6. K. S. Brown, Cohomology of groups, Springer-Verlag, New York, 1982. MR 83k:20002

7. M. Brown, A proof of the generalized Schoenflies theorem, Bull. Amer. Math. Soc. 66, 74 76 (1960). MR 22:8470b

8. R. Churney and M. Davis, When is a Coxeter system determined by its Coxeter group?, preprint (1997). 
9. F. X. Connolly and T. Koźniewski, Finiteness properties of classifying spaces of proper $\Gamma$ actions, J. of Pure and Applied Alg. 41, 17 - 36 (1986). MR 87j:57026

10. F. X. Connolly and T. Koźniewski, Rigidity and crystallographic groups I, Invent. Math. 99, 25 - 49 (1990). MR 91g:57019

11. F. X. Connolly and T. Koźniewski, Examples of lack of rigidity in crystallographic groups, in Algebraic Topology Poznań 1989, Lecture Notes in Math. no. 1474 139-145, Spinger-Verlag, New York, 1991. MR 92g:57045

12. H. S. M. Coxeter, Discrete groups generated by reflections, Ann. of Math. 35, 588 - 621 (1934).

13. M. W. Davis, Groups generated by reflections and aspherical manifolds not covered by Euclidean space, Ann. of Math. 117, 293 - 324 (1983). MR 86d:57025

14. M. W. Davis, Some aspherical manifolds, Duke Math. J. 55, 105 - 139 (1987). MR 88j:57044

15. M. W. Davis, Nonpositive curvature and reflection groups, Handbook of Geometric Topology, (eds. R. J. Daverman and R. B. Sher), Elsevier (to appear).

16. M. W. Davis, The cohomology of a Coxeter group with group ring coefficients, Duke Math. J. 91 (1998), 297-314; 95 (1998), 635. MR 99b:20067; CMP 99:06

17. V. V. Deodhar, On the root system of a Coxeter group, Comm. Algebra 10,611 - 630 (1982). MR 83j:20052a

18. A. N. Dranishnikov, On the virtual cohomological dimensions of Coxeter groups, Proc. Amer. Math. Soc. 125 (1997), 1885-1891. MR 98d:55001

19. A. Dold, Lectures on Algebraic Topology, Springer-Verlag, 1972. MR 54:3685

20. M. H. Freedman, The topology of four-dimensional manifolds, J. Diff. Geom. 17, 357 - 453 (1982). MR 84b:57006

21. M. H. Freedman and F. Quinn, Topology of 4-manifolds, Princeton University Press, Princeton, New Jersey 1990. MR 94m:57042

22. J. Harlander and H. Meinert, Higher generation subgroup sets and the virtual cohomological dimension of graph products of finite groups, J. London Math. Soc. 53, 99 - 117 (1996). MR 96m:20083

23. H. Hiller, Geometry of Coxeter Groups, Research Notes in Mathematics, No. 54, Pitman, Boston, 1982. MR 83h:14045

24. J. E. Humphreys, Reflection groups and Coxeter groups, Cambridge University Press, 1990. MR 92h:20002

25. G. Moussong, Hyperbolic Coxeter groups, Ph.D. Thesis, Ohio State University, 1988.

26. E. Rosas, Rigidity theorems for right angled reflection groups, Trans. Amer. Math. Soc. 308, 837 - 848 (1988). MR 89k:57081

27. J-P. Serre, Cohomologie des groupes discrets, in Prospects in Mathematics, Ann. Math. Studies Vol. 70, 77 - 169 (1971), Princeton University Press, Princeton. MR 54:10491

28. M. Steinberger, The equivariant topological h-cobordism theorem, Invent. Math. 91, 61 - 104 (1988). MR 89e:57015

29. M. Steinberger and J. West, Equivariant $h$-cobordisms and finiteness obstructions, Bull. Amer. Math. Soc. 12, 217 - 220 (1985). MR 86j:57019

30. E. Straume, The topological version of groups generated by reflections, Math. Z. 176, 429 446 (1981). MR 82d:20047

31. R. Strebel, A remark on subgroups of infinite index in Poincaré duality groups, Comment. Math. Helv. 52, 317 - 324 (1977). MR 56:15793

32. J. Tits, Structures et groupes de Weyl, Sém. Bourbaki (1964/65), Exp. 288, Secrétariat Mathématique, Paris, 1966. MR 33:54201 [MR 99f:00041

33. J. Tits, Sur le groupe des automorphismes de certain groupes de Coxeter, J. Algebra 113, 346-357 (1988). MR 89b:20077

34. E. B. Vinberg, Discrete linear groups generated by reflections, Math USSR Izvestija 5, 1083 - 1119 (1971). MR 46:1922

35. C. T. C. Wall, Surgery on compact manifolds, Academic Press, New York, 1970. MR 55:4217

36. S. Weinberger, The topological classification of stratified spaces, Univ. of Chicago Press, 1994. MR 96b:57024

CoordenaÇão de Pós-GraduaÇão em Matemática, Rua Mário Santos Braga, ValonGuinho Niterói, RJ 24020-005, BraziL

Division of Science and Mathematics, Birmingham-Southern College, Birmingham, Alabama 35254 